\title{
Resonant dipole-dipole interaction in the presence of dispersing and absorbing surroundings
}

\author{
Ho Trung Dungt, Ludwig Knöll, and Dirk-Gunnar Welsch \\ Theoretisch-Physikalisches Institut, Friedrich-Schiller-Universität Jena, Max-Wien-Platz 1, o7ry3 Jena, Germany
}

(May 10, 2001)

\begin{abstract}
Within the framework of quantization of the macroscopic electromagnetic field, equations of motion and an effective Hamiltonian for treating both the resonant dipole-dipole interaction between two-level atoms and the resonant atom-field interaction are derived, which can suitably be used for studying the influence of arbitrary dispersing and absorbing material surroundings on these interactions. The theory is applied to the study of the transient behavior of two atoms that initially share a single excitation, with special emphasis on the role of the two competing processes of virtual and real photon exchange in the energy transfer between the atoms. In particular, it is shown that for weak atom-field interaction there is a time window, where the energy transfer follows a rate regime of the type obtained by ordinary second-order perturbation theory. Finally, the resonant dipole-dipole interaction is shown to give rise to a doublet spectrum of the emitted light for weak atom-field interaction and a triplet spectrum for strong atom-field interaction.
\end{abstract}

PACS numbers: 42.50.Ct, 42.50.Fx 42.60.Da, 80.20.Rp

\section{INTRODUCTION}

Recently, several implementations of quantum logic gates relying on the resonant dipole-dipole interaction between two atomic qubits have been proposed. The atomic qubits could be impurity atoms in the condensed phase [1] or quantum dots embedded in a semiconductor [2]. Here and for many other applications in practice, the question of the influence of real material surroundings on the mutual interaction of the dipoles arises. Moreover, tailor-made material surroundings offer the possibility of controlling the mutual interaction of the dipoles. In particular, non-absorbing photonic crystals [3] and nonabsorbing Fabry-Pérot cavities [4,5] have been studied within the framework of mode expansion. Effects of material dispersion and absorption have been taken into account for bulk material [6], Fabry-Pérot cavities [7], and microspheres \&].

In the regime of weak atom-field coupling, the mutual resonant interaction of atoms has typically been characterized by an effective two-body potential involving atomic variables only. By analyzing a one-dimensional cavity model, it has been shown that this concept may fail to give a correct description of the interaction at least in the strong-coupling regime, where the electromagnetic field degrees of freedom can no longer be eliminated [9].
In that case, it may be instructive to treat the interaction of the atoms with the on-resonant part of the electromagnetic field exactly and the interaction with the off-resonant part in a perturbative manner. In particular, for two two-level atoms that resonantly interact with cavity-type modes, an effective Hamiltonian of the form

$$
\begin{aligned}
\hat{H}_{\mathrm{eff}}= & \hbar \sum_{\lambda} \omega_{\lambda} \hat{a}_{\lambda}^{\dagger} \hat{a}_{\lambda}+\hbar\left(\omega_{u_{A}} \hat{\sigma}_{A}^{\dagger} \hat{\sigma}_{A}+\omega_{u_{B}} \hat{\sigma}_{B}^{\dagger} \hat{\sigma}_{B}\right) \\
& +\hbar M\left(\hat{\sigma}_{A}^{\dagger} \hat{\sigma}_{B}+\hat{\sigma}_{A} \hat{\sigma}_{B}^{\dagger}\right) \\
& +\hbar \sum_{\lambda}\left(\kappa_{A \lambda} \hat{a}_{\lambda} \hat{\sigma}_{A}^{\dagger}+\kappa_{B \lambda} \hat{a}_{\lambda} \hat{\sigma}_{B}^{\dagger}+\text { H.c. }\right)
\end{aligned}
$$

has been proposed 12. Here, the first term is the energy of the cavity modes of frequencies $\omega_{\lambda}$ and photon destruction (creation) operators $\left.\hat{a}_{\lambda}\left(\hat{a}_{\lambda}^{\dagger}\right)\right]$, the second term is the energy of the two atoms, where $\hat{\sigma}_{A(B)}$ and $\hat{\sigma}_{A(B)}^{\dagger}$ are the Pauli operators of the two-level atom $A(B)$, and $\hbar \omega_{u_{A(B)}}$ is the upper-state energy, the third term is the energy of the so-called resonant dipole-dipole interaction of the atoms to each other ( $\hbar M$ - coupling strength), and the fourth term is the familiar interaction energy between the atoms and the cavity modes in the rotatingwave approximation. Apart from the fact that the coupling parameters in the Hamiltonian (1) are not specified and their relation to each other thus remains unclear (also see 10,11), material absorption cannot be taken into account, because of the underlying concept of mode decomposition.

In this paper we give a rigorous derivation of an effective Hamiltonian, which - although at first glance looks like that in Eq. (11) - applies to atoms in arbitrary dispersing and absorbing material surroundings, with the coupling parameters being well defined. For this purpose, we start from the multipolar Hamiltonian governing the motion of the coupled system of two-level atoms and the medium-assisted electromagnetic field, with the medium being described in terms of a spatially varying permittivity that is a complex function of frequency (for a review, see 13]). The quantity that essentially governs the strength of the atom-field interaction is the (classical) Green tensor of the inhomogeneous Helmholtz equation with the space- and frequency-dependent complex permittivity of the material surroundings. Treating the off-resonant part of the atom-field interaction in a (coarse-grained) Markov approximation, whereas the on-resonant part of the atom-field interaction is left in the original form, we derive the density-matrix equations 
of motion for the coupled atom-field system, where the off-resonant atom-field interaction is eliminated in favor of the resonant dipole-dipole interaction. We then show that under certain conditions an effective Hamiltonian that governs the motion can be constructed. The results show that the resonant dipole-dipole interaction (via the off-resonant atom-field interaction) and the on-resonant atom-field interaction, respectively, are essentially determined by the real and the imaginary part of the mediumassisted Green tensor. Whereas the spontaneous decay is only determined by the imaginary part, the mutual interaction of the atoms is determined by both the real and the imaginary part of the Green tensor.

To illustrate the theory, we examine, for both weak and strong atom-field interaction, the temporal evolution of two two-level atoms that initially share a single excitation, with special emphasis on the interatomic energy transfer. In particular for weak atom-field interaction, from the exact time dependence of the upper-state population of the acceptor atom we infer an energy transfer rate. We show that it is essentially the same rate as the commonly used rate, i.e., the transition probability per unit time which is obtained by means of Fermi's golden rule in second-order perturbation theory with regard to the original Hamiltonian. Finally we address the problem of the influence of the resonant dipole-dipole interaction on the spectrum of the light emitted by the atoms in the limits of weak and strong atom-field interaction.

The paper is organized as follows. In Section II the density-matrix equations of motion for the system that consists of the atoms and the resonant part of the electromagnetic field are derived. The problem of deriving them from an effective Hamiltonian is studied in Section III. The temporal evolution of two atoms that initially share a single excitation is studied in Section IV. The problem of determining an energy transfer rate is considered in Section Q, and Section VI is devoted to the spectral properties of the emitted light. Finally, a summary and some concluding remarks are given in Section VII.

\section{DENSITY-MATRIX EQUATION}

Let us consider $N$ two-level atoms [positions $\mathbf{r}_{A}$, transition frequencies $\omega_{A}$, dipole moments $\mathbf{d}_{A}(A=1,2, \ldots, N)$ that resonantly interact with the electromagnetic field via electric-dipole transitions in the presence of dispersing and absorbing bodies. The corresponding multipolarcoupling Hamiltonian reads as 13, 14,

$$
\begin{gathered}
\hat{H}=\int \mathrm{d}^{3} \mathbf{r} \int_{0}^{\infty} \mathrm{d} \omega \hbar \omega \hat{\mathbf{f}}^{\dagger}(\mathbf{r}, \omega) \hat{\mathbf{f}}(\mathbf{r}, \omega)+\sum_{A} \frac{1}{2} \hbar \omega_{A} \hat{\sigma}_{A z} \\
\quad-\sum_{A} \int_{0}^{\infty} \mathrm{d} \omega\left[\hat{\mathbf{d}}{ }_{A} \underline{\hat{\mathbf{E}}}\left(\mathbf{r}_{A}, \omega\right)+\text { H.c. }\right]
\end{gathered}
$$

where

$$
\hat{\mathbf{d}}_{A}=\mathbf{d}_{A} \hat{\sigma}_{A}+\mathbf{d}_{A}^{*} \hat{\sigma}_{A}^{\dagger}
$$

and

$$
\underline{\hat{\mathbf{E}}}(\mathbf{r}, \omega)=i \sqrt{\frac{\hbar}{\pi \varepsilon_{0}}} \frac{\omega^{2}}{c^{2}} \int \mathrm{d}^{3} \mathbf{r}^{\prime} \sqrt{\varepsilon_{\mathrm{I}}\left(\mathbf{r}^{\prime}, \omega\right)} \boldsymbol{G}\left(\mathbf{r}, \mathbf{r}^{\prime}, \omega\right) \hat{\mathbf{f}}\left(\mathbf{r}^{\prime}, \omega\right) .
$$

Here, $\hat{\mathbf{f}}(\mathbf{r}, \omega)$ and $\hat{\mathbf{f}}^{\dagger}(\mathbf{r}, \omega)$ are bosonic fields which play the role of the fundamental variables of the electromagnetic field and the medium, including a reservoir necessarily associated with the losses in the medium, $\boldsymbol{G}\left(\mathbf{r}, \mathbf{r}^{\prime}, \omega\right)$ is the classical Green tensor, and $\varepsilon(\mathbf{r}, \omega)=\varepsilon_{\mathrm{R}}(\mathbf{r}, \omega)+i \varepsilon_{\mathrm{I}}(\mathbf{r}, \omega)$ is the complex (Kramers-Kronig consistent) permittivity. It should be pointed out that there are no direct Coulomb forces between particles in the Hamiltonian (2); all interactions are mediated by the medium-assisted electromagnetic field.

With regard to the interaction of the atoms with the electromagnetic field, it is convenient to decompose the latter into an on-resonant part (denoted by $\int_{0}^{\prime \infty} \mathrm{d} \omega \ldots$ ) and an off-resonant part (denoted by $\int_{0}^{\prime \prime \infty} \mathrm{d} \omega \ldots$... Let us now consider the temporal evolution of the system that consists of the atoms and the on-resonant part of the electromagnetic field. If $\hat{O}$ is any system operator, we may write its equation of motion in the Heisenberg picture as, on recalling the Hamiltonian (2),

$$
\begin{aligned}
\dot{\hat{O}}=- & \frac{i}{\hbar}\left[\hat{O}, \hat{H}_{\mathrm{S}}\right] \\
+ & \frac{i}{\hbar} \sum_{A} \int_{0}^{\prime \prime \infty} \mathrm{d} \omega\left\{\left[\hat{O}, \hat{\mathbf{d}}_{A}\right] \underline{\hat{\mathbf{E}}}\left(\mathbf{r}_{A}, \omega\right)\right. \\
& \left.+\underline{\hat{\mathbf{E}}}^{\dagger}\left(\mathbf{r}_{A}, \omega\right)\left[\hat{O}, \hat{\mathbf{d}}_{A}\right]\right\},
\end{aligned}
$$

where

$$
\begin{gathered}
\hat{H}_{\mathrm{S}}=\int \mathrm{d}^{3} \mathbf{r} \int_{0}^{\prime \infty} \mathrm{d} \omega \hbar \omega \hat{\mathbf{f}}^{\dagger}(\mathbf{r}, \omega) \hat{\mathbf{f}}(\mathbf{r}, \omega)+\sum_{A} \frac{1}{2} \hbar \omega_{A} \hat{\sigma}_{A z} \\
-\sum_{A} \int_{0}^{\prime \infty} \mathrm{d} \omega\left[\hat{\mathbf{d}} \hat{\mathbf{E}}\left(\mathbf{r}_{A}, \omega\right)+\text { H.c. }\right] .
\end{gathered}
$$

Note that in Eq. (5) normal ordering is adopted such that $\underline{\hat{\mathbf{E}}}\left(\mathbf{r}_{A}, \omega\right)$ is on the right-hand side and $\underline{\mathbf{E}}^{\dagger}\left(\mathbf{r}_{A}, \omega\right)$ is on the left-hand side in operator products. According to Eq. (雨), the operators $\underline{\hat{\mathbf{E}}}\left(\mathbf{r}_{A}, \omega\right)$ and $\underline{\hat{\mathbf{E}}}^{\dagger}\left(\mathbf{r}_{A}, \omega\right)$ in Eq. (5) are thought to be expressed in terms of the basic-variable operators $\hat{\mathbf{f}}(\mathbf{r}, \omega)$ and $\hat{\mathbf{f}}^{\dagger}(\mathbf{r}, \omega)$ respectively. It is not difficult to see that $\hat{\mathbf{f}}(\mathbf{r}, \omega)$ obeys the Heisenberg equation of motion

$$
\begin{aligned}
\dot{\hat{\mathbf{f}}}(\mathbf{r}, \omega)= & -i \omega \hat{\mathbf{f}}(\mathbf{r}, \omega) \\
& +\frac{\omega^{2}}{c^{2}} \sqrt{\frac{\varepsilon_{\mathrm{I}}(\mathbf{r}, \omega)}{\hbar \pi \varepsilon_{0}}} \sum_{A} \hat{\mathbf{d}}_{A} \boldsymbol{G}^{*}\left(\mathbf{r}_{A}, \mathbf{r}, \omega\right) .
\end{aligned}
$$


We now solve Eq. (7) formally, insert the result into Eq. (5), apply a (coarse-grained) Markov approximation to the slowly varying atomic variables in the time integrals in the off-resonant frequency integrals, and assume that the off-resonant free-field is initially $(t=0)$ prepared in the vacuum state. After some algebra, we arrive at the following equation of motion for the expectation value of the system operator $\hat{O}$ (Appendix A):

$$
\begin{aligned}
& \langle\dot{\hat{O}}\rangle=-\frac{i}{\hbar}\left\langle\left[\hat{O}, \hat{\tilde{H}}_{\mathrm{S}}\right]\right\rangle \\
& +i \sum_{A, A^{\prime}}^{\prime}\left\{\delta_{A^{*} A^{\prime}}^{-}\left\langle\left[\hat{O}, \hat{\sigma}_{A}^{\dagger}\right] \hat{\sigma}_{A^{\prime}}\right\rangle+\delta_{A A^{\prime *}}^{+}\left\langle\left[\hat{O}, \hat{\sigma}_{A}\right] \hat{\sigma}_{A^{\prime}}^{\dagger}\right\rangle\right. \\
& \left.\quad+\delta_{A A^{\prime *}}^{-}\left\langle\hat{\sigma}_{A^{\prime}}^{\dagger}\left[\hat{O}, \hat{\sigma}_{A}\right]\right\rangle+\delta_{A^{*} A^{\prime}}^{+}\left\langle\hat{\sigma}_{A^{\prime}}\left[\hat{O}, \hat{\sigma}_{A}^{\dagger}\right]\right\rangle\right\},
\end{aligned}
$$

where the notation $\sum_{A, A^{\prime}}^{\prime}$ indicates that $A \neq A^{\prime}$. In particular, when $\hat{O}$ is identified with the atomic operator $\hat{\sigma}_{A}$, Eq. (8) yields

$$
\left\langle\dot{\hat{\sigma}}_{A}\right\rangle=-\frac{i}{\hbar}\left\langle\left[\hat{\sigma}_{A}, \hat{\tilde{H}}_{\mathrm{S}}\right]\right\rangle-i \sum_{\substack{A^{\prime} \\ A^{\prime} \neq A}} \delta_{A^{*} A^{\prime}}\left\langle\hat{\sigma}_{A z} \hat{\sigma}_{A^{\prime}}\right\rangle .
$$

In Eqs. (8) and (9), the system Hamiltonian $\hat{\tilde{H}}_{\mathrm{S}}$ is defined according to Eq. (6), with $\omega_{A}$ being replaced by $\tilde{\omega}_{A}$, where

$$
\begin{gathered}
\tilde{\omega}_{A}=\omega_{A}-\delta_{A^{*} A}, \\
\delta_{A^{*} A}=\delta_{A^{*} A}^{-}-\delta_{A^{*} A}^{+}, \\
\delta_{A A}^{-(+)}=\frac{\mathcal{P}}{\pi \hbar \varepsilon_{0}} \int_{0}^{\infty} \mathrm{d} \omega \frac{\omega^{2}}{c^{2}} \frac{\mathbf{d}_{A} \operatorname{Im} \boldsymbol{G}\left(\mathbf{r}_{A}, \mathbf{r}_{A}, \omega\right) \mathbf{d}_{A}}{\omega-(+) \omega_{A}},
\end{gathered}
$$

and the resonant interatomic coupling parameters are defined according to

$$
\left.\delta_{A^{*} A^{\prime}}\right|_{A \neq A^{\prime}}=\delta_{A^{*} A^{\prime}}^{-}+\delta_{A^{*} A^{\prime}}^{+},
$$

$$
\begin{aligned}
& \left.\delta_{A A^{\prime}}^{-(+)}\right|_{A \neq A^{\prime}} \\
& =\frac{\mathcal{P}}{\pi \hbar \varepsilon_{0}} \int_{0}^{\infty} \mathrm{d} \omega \frac{\omega^{2}}{c^{2}} \frac{\mathbf{d}_{A} \operatorname{Im} \boldsymbol{G}\left(\mathbf{r}_{A}, \mathbf{r}_{A^{\prime}}, \omega\right) \mathbf{d}_{A^{\prime}}}{\omega-(+) \tilde{\omega}_{A^{\prime}}}
\end{aligned}
$$

$\left[\mathcal{P}\right.$ - principal value]. The notation $A^{*}\left(A^{\prime *}\right)$ means that $\mathbf{d}_{A}\left(\mathbf{d}_{A^{\prime}}\right)$ in Eqs. (12) and (14) has to be replaced with its complex conjugate $\mathbf{d}_{A}^{*}\left(\mathbf{d}_{A^{\prime}}^{*}\right)$. Applying the KramersKronig relation to the Green tensor, from Eq. (14) we derive that

$$
\delta_{A A^{\prime}}^{-}=\frac{\tilde{\omega}_{A^{\prime}}^{2}}{\hbar \varepsilon_{0} c^{2}} \mathbf{d}_{A} \operatorname{Re} \boldsymbol{G}\left(\mathbf{r}_{A}, \mathbf{r}_{A^{\prime}}, \tilde{\omega}_{A^{\prime}}\right) \mathbf{d}_{A^{\prime}}-\delta_{A A^{\prime}}^{+},
$$

and Eq. (13) thus becomes

$$
\left.\delta_{A^{*} A^{\prime}}\right|_{A \neq A^{\prime}}=\frac{\tilde{\omega}_{A^{\prime}}^{2}}{\hbar \varepsilon_{0} c^{2}} \mathbf{d}_{A}^{*} \operatorname{Re} \boldsymbol{G}\left(\mathbf{r}_{A}, \mathbf{r}_{A^{\prime}}, \tilde{\omega}_{A^{\prime}}\right) \mathbf{d}_{A^{\prime}} .
$$

Accordingly, from Eqs. (12) and (11) it follows that

$$
\delta_{A^{*} A}=\frac{\omega_{A}^{2}}{\hbar \varepsilon_{0} c^{2}} \mathbf{d}_{A}^{*} \operatorname{Re} \boldsymbol{G}\left(\mathbf{r}_{A}, \mathbf{r}_{A}, \omega_{A}\right) \mathbf{d}_{A}-2 \delta_{A^{*} A}^{+} .
$$

In dealing with problems of atoms embedded in media, the atoms should be assumed to be localized in some small free-space regions, so that the Green tensor at the positions of the atoms can always be written as a sum of the vacuum Green tensor $G^{V}$ and the reflection Green tensor $\boldsymbol{G}^{R}$. Due to the singularity of $\operatorname{Re} \boldsymbol{G}^{V}$ at equal space points, Eq. (17) actually applies to the reflection part only. The vacuum part, calculated in many textbooks, can be thought of as being already included in $\omega_{A}$.

It should be pointed out that the single-atom frequency shift $\delta_{A^{*} A}$ [Eq. (11) or Eq. (17)] (which is a real quantity because of the reciprocity property of the Green tensor) differs from the frequency shift $\delta_{A^{*} A}^{-}$obtained in the rotating-wave approximation (see, e.g., Ref. [15]) in the term $\delta_{A^{*} A}^{+}$, which results from the counter-rotating contributions to the Hamiltonian. Note that when the atoms are in free space, then Eqs. (11) and (13) reduce to the result derived in Refs. 16 20 . Since the first term on the right-hand side in Eq. (17) can also be obtained classically [21], the second term $\delta_{A^{*} A}^{+}$is sometimes termed the quantum correction. It is interesting to note that this quantum correction just corrects the rotating-wave result. For that part of the frequency shift which is caused by the presence of the macroscopic bodies (mathematically, by the reflection part of the Green tensor), the quantum correction may safely be neglected.

From Eq. (16) it is seen that when the transition frequencies $\tilde{\omega}_{A}$ and $\tilde{\omega}_{A^{\prime}}$ of two atoms $A$ and $A^{\prime}$ are different from each other, then the strengths of the resonant dipole-dipole coupling $\left|\delta_{A^{*} A^{\prime}}\right|$ and $\left|\delta_{A^{\prime *} A}\right|$ are not symmetric with respect to $A$ and $A^{\prime}$. Only if the differences $\left|\tilde{\omega}_{A}-\tilde{\omega}_{A^{\prime}}\right|$ are small compared with the frequency scale of variation of the Green tensor, this asymmetry can be disregarded and $\tilde{\omega}_{A}$ and $\tilde{\omega}_{A^{\prime}}$ may be replaced by an appropriately chosen mid-frequency, say $\left(\tilde{\omega}_{A}+\tilde{\omega}_{A^{\prime}}\right) / 2$. Whereas for atoms in free space such an approximation is unproblematic even for relatively large frequency differences, the situation can drastically change if the presence of macroscopic bodies gives rise to a highly peaked Green tensor that rapidly varies with frequency.

Recalling the relationship

$$
\begin{aligned}
\langle\hat{O}(t)\rangle & =\operatorname{Tr}[\hat{\rho}(0) \hat{O}(t)] \\
& =\operatorname{Tr}[\hat{\rho}(t) \hat{O}(0)]=\operatorname{Tr}[\hat{\varrho}(t) \hat{O}(0)],
\end{aligned}
$$

where $\hat{O}$ is an arbitrary system operator, $\hat{\rho}$ is the density operator of the overall system, and $\varrho$ is the (reduced) density operator of the system, and making use of the cyclic properties of the trace, from Eq. (8) we derive the 
following equation of motion for the system density operator in the Schrödinger picture (Appendix A):

$$
\begin{aligned}
\dot{\hat{\varrho}}=- & \frac{i}{\hbar}\left[\hat{\tilde{H}}_{\mathrm{S}}, \hat{\varrho}\right] \\
+ & \left\{i \sum _ { A , A ^ { \prime } } ^ { \prime } \left[\delta_{A^{*} A^{\prime}}^{-}\left(\hat{\sigma}_{A}^{\dagger} \hat{\sigma}_{A^{\prime}} \hat{\varrho}-\hat{\sigma}_{A^{\prime}} \hat{\varrho} \hat{\sigma}_{A}^{\dagger}\right)\right.\right. \\
& \left.\left.+\delta_{A A^{\prime *}}^{+}\left(\hat{\sigma}_{A} \hat{\sigma}_{A^{\prime}}^{\dagger} \hat{\varrho}-\hat{\sigma}_{A^{\prime}}^{\dagger} \hat{\varrho} \hat{\sigma}_{A}\right)\right]+ \text { H.c. }\right\} .
\end{aligned}
$$

In this equation both the resonant interatomic interaction and the resonant atom-field interaction are taken into account, without any restriction to the strength of the latter one. It is worth noting that this type of equation cannot be derived from an effective Hamiltonian in general.

In particular in the case when the resonant atom-field interaction is sufficiently weak, it can also be treated in a Markov approximation. Let $\varrho$ be the reduced density operator of the atomic system and let us assume that the on-resonant part of the electromagnetic field is initially also prepared in the vacuum state (i.e., there is no external driving field). By tracing out both the on- and the off- resonant medium-assisted field variables, we arrive at the following master equation for the reduced density operator of the atomic system (see Appendix B):

$$
\begin{aligned}
\dot{\hat{\varrho}}= & -\frac{1}{2} i \sum_{A} \tilde{\omega}_{A}\left[\hat{\sigma}_{A z}, \hat{\varrho}\right] \\
- & \frac{1}{2}\left[\sum_{A, A^{\prime}} \Gamma_{A^{*} A^{\prime}}\left(\hat{\sigma}_{A}^{\dagger} \hat{\sigma}_{A^{\prime}} \hat{\varrho}-\hat{\sigma}_{A^{\prime}} \hat{\varrho} \hat{\sigma}_{A}^{\dagger}\right)+\text { H.c. }\right] \\
+ & \left\{i \sum _ { A , A ^ { \prime } } ^ { \prime } \left[\delta_{A^{*} A^{\prime}}^{-}\left(\hat{\sigma}_{A}^{\dagger} \hat{\sigma}_{A^{\prime}} \hat{\varrho}-\hat{\sigma}_{A^{\prime}} \varrho \hat{\sigma}_{A}^{\dagger}\right)\right.\right. \\
& \left.\left.+\delta_{A A^{\prime *}}^{+}\left(\hat{\sigma}_{A^{\prime}} \hat{\sigma}_{A^{\prime}}^{\dagger} \hat{\varrho}-\hat{\sigma}_{A^{\prime}}^{\dagger} \hat{\varrho} \hat{\sigma}_{A}\right)\right]+ \text { H.c. }\right\},
\end{aligned}
$$

where

$$
\Gamma_{A A^{\prime}}=\frac{2 \tilde{\omega}_{A^{\prime}}^{2}}{\hbar \varepsilon_{0} c^{2}} \mathbf{d}_{A} \operatorname{Im} \boldsymbol{G}\left(\mathbf{r}_{A}, \mathbf{r}_{A^{\prime}}, \tilde{\omega}_{A^{\prime}}\right) \mathbf{d}_{A^{\prime}} .
$$

If the differences between the atomic transition frequencies are small in comparison with the frequency scale of variation of the Green tensor, so that the relations

$$
\delta_{A^{*} A^{\prime}}^{ \pm} \simeq \delta_{A^{\prime} A^{*}}^{ \pm}
$$

and

$$
\Gamma_{A^{*} A^{\prime}} \simeq \Gamma_{A^{\prime} A^{*}}
$$

are valid, then Eq. (20) reduces to

$$
\begin{array}{r}
\dot{\hat{\varrho}}=-\frac{1}{2} i \sum_{A} \tilde{\omega}_{A}\left[\hat{\sigma}_{A z}, \hat{\varrho}\right]+i \sum_{A, A^{\prime}}^{\prime} \delta_{A^{*} A^{\prime}}\left[\hat{\sigma}_{A}^{\dagger} \hat{\sigma}_{A^{\prime}}, \hat{\varrho}\right] \\
-\frac{1}{2} \sum_{A, A^{\prime}} \Gamma_{A^{*} A^{\prime}}\left(\hat{\sigma}_{A}^{\dagger} \hat{\sigma}_{A^{\prime}} \hat{\varrho}-2 \hat{\sigma}_{A^{\prime}} \varrho \hat{\sigma}_{A}^{\dagger}+\hat{\varrho} \hat{\sigma}_{A^{\dagger}}^{\dagger} \hat{\sigma}_{A^{\prime}}\right),
\end{array}
$$

which is of the same form as the master equation obtained on the basis of Kubo's formula for the field correlation functions [7].

\section{EFFECTIVE HAMILTONIAN}

Let us return to Eqs. (8) and (19) and restrict our attention to the case when the difference between the atomic transition frequencies is small in comparison with the frequency scale of variation of the Green tensor, so that Eq. (22) holds. Then Eqs. (8) and (19) reduce to

$$
\langle\dot{\hat{O}}\rangle=-\frac{i}{\hbar}\left\langle\left[\hat{O},\left(\hat{\tilde{H}}_{\mathrm{S}}-\sum_{A, A^{\prime}}^{\prime} \hbar \delta_{A^{*} A^{\prime}} \hat{\sigma}_{A}^{\dagger} \hat{\sigma}_{A^{\prime}}\right)\right]\right\rangle
$$

and

$$
\dot{\hat{\varrho}}=-\frac{i}{\hbar}\left[\left(\hat{\tilde{H}}_{\mathrm{S}}-\sum_{A, A^{\prime}}^{\prime} \hbar \delta_{A^{*} A^{\prime}} \hat{\sigma}_{A}^{\dagger} \hat{\sigma}_{A^{\prime}}\right), \hat{\varrho}\right],
$$

respectively. Recalling the definition of $\hat{\tilde{H}}_{S}$, we see that the motion of the system is governed by the effective Hamiltonian

$$
\begin{aligned}
\hat{H}_{\mathrm{eff}}= & \int \mathrm{d}^{3} \mathbf{r} \int_{0}^{\prime \infty} \mathrm{d} \omega \hbar \omega \hat{\mathbf{f}}^{\dagger}(\mathbf{r}, \omega) \hat{\mathbf{f}}(\mathbf{r}, \omega) \\
& +\sum_{A} \frac{1}{2} \hbar \tilde{\omega}_{A} \hat{\sigma}_{A z}-\sum_{A, A^{\prime}}^{\prime} \hbar \delta_{A^{*} A^{\prime}} \hat{\sigma}_{A}^{\dagger} \hat{\sigma}_{A^{\prime}} \\
& -\sum_{A} \int_{0}^{\prime \infty} \mathrm{d} \omega\left[\hat{\mathbf{d}}_{A} \hat{\hat{\mathbf{E}}}\left(\mathbf{r}_{A}, \omega\right)+\text { H.c. }\right],
\end{aligned}
$$

with $\underline{\hat{\mathbf{E}}}\left(\mathbf{r}_{A}, \omega\right)$ being given by Eq. (4).

From the above it is clear that a Hamiltonian of the type (11) makes only sense if the atomic transition frequencies are sufficiently near to each other. In that case, the Hamiltonian (27) is the desired extension of the Hamiltonian (11). The Hamiltonian (27) is remarkable in several respects. Firstly, it applies to atoms surrounded by arbitrarily configured, dispersive and absorptive media. Secondly, it goes beyond the rotating-wave approximation. Thirdly, it contains a resonant dipole-dipole coupling energy that is explicitly expressed in terms of the medium-assisted Green tensor, according to Eq. (16).

The first term in Eq. 27) describes, as before, the free medium-assisted electromagnetic field energy. The second term is the energy of the free atoms, which takes account of the medium-induced single-atom transition frequency shift. As already mentioned, the third term 
represents the energy of the resonant dipole-dipole interaction of the atoms. Note that both the single-atom transition frequency shift and the interatom resonant dipoledipole interaction result from the off-resonant atom-field coupling. The energy of the resonant interaction of the atoms with the electromagnetic field is given by the fourth term, which is of course not only responsible, e.g., for the single-atom spontaneous decay in the regime of weak atom-field coupling and the Rabi-type oscillations in the strong-coupling regime, but it also determines, together with the resonant dipole-dipole coupling, the mutual interaction of the atoms. In particular, in the limit of a $\delta$-like field excitation the atoms are resonantly coupled to, the fourth term in Eq. 27) reduces to the interaction energy in the Tavis-Cummings model [22. Note that within the Tavis-Cummings model the resonant dipole-dipole coupling between the atoms cannot be described, because this model does not take account of the off-resonant atom-field interaction. In practice the excitation spectrum of any real electromagnetic field is always continuous, and the inclusion in the Hamiltonian of the off-resonant atom-field interaction is thus quite crucial, because it may lead to observable effects.

It is worth noting that, as can be seen from Eq. (16), the strength of the resonant dipole-dipole interaction is determined by the real part of the Green tensor at different space points. Thus, it is affected by a surrounding medium in a quite different way as the spectral excitation density of the medium-assisted electromagnetic field, which is determined by the imaginary part of the Green tensor at equal space points. In particular, it may happen that a high (low) excitation density and thus an enhanced (reduced) single-atom decay rate is accompanied by a reduced (enhanced) strength of the resonant dipole-dipole coupling [14].

Clearly, strong resonant dipole-dipole interaction can only be expected if the atoms are sufficiently close to each other. To give a simple example of the effect of material absorption, let us assume that the atoms are embedded in bulk material of complex permittivity $\varepsilon(\omega)$. Using the bulk-material Green tensor (see, e.g., [13]), from Eq. (16) we find in the short-distance limit

$$
\delta_{A^{*} A^{\prime}}=\frac{1}{4 \pi \hbar \varepsilon_{0} R^{3}} \operatorname{Re}\left[\frac{1}{\varepsilon\left(\tilde{\omega}_{A^{\prime}}\right)}\right]\left(3 \frac{\mathbf{d}_{A}^{*} \mathbf{R}}{R} \frac{\mathbf{d}_{A^{\prime}} \mathbf{R}}{R}-\mathbf{d}_{A}^{*} \mathbf{d}_{A^{\prime}}\right)
$$

$\left(\mathbf{R}=\mathbf{r}_{A}-\mathbf{r}_{A^{\prime}}\right)$. In free space, Eq. (28) reduces to the wellknown result that the resonant dipole-dipole coupling simply corresponds to the (near-field) Coulomb-type interaction. It is seen that the characteristic $R^{-3}$ distance dependence observed in free space is not changed by the medium. In the long-distance limit, we find that

$$
\begin{array}{r}
\delta_{A^{*} A^{\prime}}=\frac{c^{2}}{4 \pi \hbar \varepsilon_{0} R \tilde{\omega}_{A^{\prime}}^{2}}\left(\mathbf{d}_{A}^{*} \mathbf{d}_{A^{\prime}}-\frac{\mathbf{d}_{A}^{*} \mathbf{R}}{R} \frac{\mathbf{d}_{A^{\prime}} \mathbf{R}}{R}\right) \\
\times \cos \left[n_{\mathrm{R}}\left(\tilde{\omega}_{A^{\prime}}\right) \frac{\tilde{\omega}_{A^{\prime}} R}{c}\right] \exp \left[-n_{\mathrm{I}}\left(\tilde{\omega}_{A^{\prime}}\right) \frac{\tilde{\omega}_{A^{\prime}} R}{c}\right]
\end{array}
$$

$\left[n(\omega)=n_{\mathrm{R}}(\omega)+i n_{\mathrm{I}}(\omega)=\sqrt{\varepsilon(\omega)}\right]$, i.e., the (harmonically modulated) $R^{-1}$ dependence observed in free space is changed to an exponential decrease according to $\exp \left[-n_{\mathrm{I}}\left(\tilde{\omega}_{A^{\prime}}\right) \tilde{\omega}_{A^{\prime}} R / c\right]$ due to material absorption. Thus, material absorption can drastically reduce the resonant dipole-dipole coupling strength with increasing mutual distance of the atoms.

\section{TEMPORAL EVOLUTION OF A TWO-ATOM SYSTEM}

Let us use the effective Hamiltonian (27) to study a system of two-level atoms (resonantly) coupled to the medium-assisted electromagnetic field and assume that initially the atoms share a single excitation while the field is in the vacuum state. In the Schrödinger picture we may write, on omitting off-resonant terms, the state vector of the system in the form of

$$
\begin{aligned}
& |\psi(t)\rangle=\sum_{A} C_{A}(t) e^{-i\left(\tilde{\omega}_{A}-\bar{\omega}\right) t}\left|U_{A}\right\rangle|\{0\}\rangle \\
& +\int \mathrm{d}^{3} \mathbf{r} \int_{0}^{\prime \infty} \mathrm{d} \omega C_{L i}(\mathbf{r}, \omega, t) e^{-i(\omega-\bar{\omega}) t}|L\rangle \hat{f}_{i}^{\dagger}(\mathbf{r}, \omega)|\{0\}\rangle
\end{aligned}
$$

$\left(\bar{\omega}=\frac{1}{2} \sum_{A} \tilde{\omega}_{A}\right)$. Here, $\left|U_{A}\right\rangle$ is the atomic state with the $A$ th atom in the upper state and all the other atoms in the lower state, and $|L\rangle$ is the atomic state with all atoms in the lower state. Accordingly, $|\{0\}\rangle$ is the vacuum state of the rest of the system, and $\hat{f}_{i}^{\dagger}(\mathbf{r}, \omega)|\{0\}\rangle$ is the state, where a single quantum is excited.

From the Hamiltonian (27), the equations of motion for the slowly varying probability amplitudes $C_{A}$ and $\mathbf{C}_{L}$ read as

$$
\begin{aligned}
& \dot{C}_{A}(t)=\sum_{\substack{A^{\prime} \\
A^{\prime} \neq A}} i \delta_{A^{*} A^{\prime}} e^{i\left(\tilde{\omega}_{A}-\tilde{\omega}_{A^{\prime}}\right) t} C_{A^{\prime}}(t) \\
&- \frac{1}{\sqrt{\pi \epsilon_{0} \hbar}} \int_{0}^{\prime \infty} \mathrm{d} \omega \frac{\omega^{2}}{c^{2}} \int \mathrm{d}^{3} \mathbf{r}\left[\sqrt{\varepsilon_{\mathrm{I}}(\mathbf{r}, \omega)}\right. \\
&\left.\times \mathbf{d}_{A}^{*} \boldsymbol{G}\left(\mathbf{r}_{A}, \mathbf{r}, \omega\right) \mathbf{C}_{L}(\mathbf{r}, \omega, t) e^{-i\left(\omega-\tilde{\omega}_{A}\right) t}\right] \\
& \dot{\mathbf{C}}_{L}(\mathbf{r}, \omega, t)=\frac{1}{\sqrt{\pi \epsilon_{0} \hbar}} \frac{\omega^{2}}{c^{2}} \sqrt{\varepsilon_{\mathrm{I}}(\mathbf{r}, \omega)} \\
& \times \sum_{A^{\prime}} \mathbf{d}_{A^{\prime}} \boldsymbol{G}^{*}\left(\mathbf{r}_{A^{\prime}}, \mathbf{r}, \omega\right) C_{A^{\prime}}(t) e^{i\left(\omega-\tilde{\omega}_{A^{\prime}}\right) t} .
\end{aligned}
$$

By formally integrating Eq. (32) under the initial condition that $\mathbf{C}_{L}(t=0)=0$, and substituting the formal solution into Eq. (31), we obtain the following system of integrodifferential equations for the $C_{A}$ :

$$
\dot{C}_{A}(t)=\sum_{\substack{A^{\prime} \\ A^{\prime} \neq A}} i \delta_{A^{*} A^{\prime}} e^{i\left(\tilde{\omega}_{A}-\tilde{\omega}_{A^{\prime}}\right) t} C_{A^{\prime}}(t)
$$




$$
+\sum_{A^{\prime}} \int_{0}^{t} \mathrm{~d} t^{\prime} \int_{0}^{\prime \infty} \mathrm{d} \omega K_{A^{*} A^{\prime}}\left(t, t^{\prime} ; \omega\right) C_{A^{\prime}}\left(t^{\prime}\right),
$$

where

$$
\begin{aligned}
K_{A A^{\prime}}\left(t, t^{\prime} ; \omega\right)=-\frac{1}{\hbar \pi \varepsilon_{0}}\left[\frac{\omega^{2}}{c^{2}} e^{-i\left(\omega-\tilde{\omega}_{A}\right) t} e^{i\left(\omega-\tilde{\omega}_{A^{\prime}}\right) t^{\prime}}\right. \\
\left.\times \mathbf{d}_{A} \operatorname{Im} \boldsymbol{G}\left(\mathbf{r}_{A}, \mathbf{r}_{A^{\prime}}, \omega\right) \mathbf{d}_{A^{\prime}}\right] .
\end{aligned}
$$

It should be pointed out that equations of the type (30) - (34) can also be derived on the basis of the original Hamiltonian (2) in the rotating wave approximation $\left(\int_{0}^{\infty} \mathrm{d} \omega \ldots \rightarrow \int_{0}^{\infty} \mathrm{d} \omega \ldots\right)$, without the restrictive condition that the Green tensor does not change on a frequency scale defined by the differences of the atomic transition frequencies [8]. Clearly, in such an approach, the contributions of the counter-rotating terms to the frequency shifts and interatomic coupling strengths are disregarded. In order to get insight into the atomic motion on the basis of closed solutions of Eq. (33), let us consider two atoms and restrict our attention to the limiting cases of weak and strong atom-field coupling.

\section{A. Weak atom-field coupling}

In the weak coupling regime, the integral expression in Eq. (33) can be treated in a (coarse-grained) Markov approximation, i.e., $C_{A^{\prime}}\left(t^{\prime}\right)$ is replaced by $C_{A^{\prime}}(t)$, and the time integral $\int_{0}^{t} \mathrm{~d} t^{\prime} e^{-i\left(\omega-\tilde{\omega}_{A^{\prime}}\right)\left(t-t^{\prime}\right)}$ is replaced by the $\zeta$-function $\zeta\left(\tilde{\omega}_{A^{\prime}}-\omega\right)$. Since the frequency integral only runs over the resonance region, the $\zeta$-function effectively acts as the $\delta$-function, and we arrive at the following equations for the (slowly-varying) upper-state probability amplitudes of two atoms $A$ and $B$ :

$$
\begin{aligned}
& \dot{C}_{A}(t)=-\frac{1}{2} \Gamma_{A^{*} A} C_{A}(t)+\mathcal{K}_{A^{*} B} e^{i\left(\tilde{\omega}_{A}-\tilde{\omega}_{B}\right) t} C_{B}(t), \\
& \dot{C}_{B}(t)=-\frac{1}{2} \Gamma_{B^{*} B} C_{B}(t)+\mathcal{K}_{B^{*} A} e^{-i\left(\tilde{\omega}_{A}-\tilde{\omega}_{B}\right) t} C_{A}(t),
\end{aligned}
$$

where

$$
\begin{aligned}
\mathcal{K}_{A B} & =-\frac{1}{2} \Gamma_{A B}+i \delta_{A B} \\
& =i \frac{\tilde{\omega}_{B}^{2}}{\hbar \varepsilon_{0} c^{2}} \mathbf{d}_{A} \boldsymbol{G}\left(\mathbf{r}_{A}, \mathbf{r}_{B}, \tilde{\omega}_{B}\right) \mathbf{d}_{B} .
\end{aligned}
$$

We now make the simplifying assumption that the transition frequencies of the two atoms are nearly equal to each other, $\tilde{\omega}_{A} \simeq \tilde{\omega}_{B}$, but allow for $\Gamma_{A^{*} A} \neq \Gamma_{B^{*} B}$. The latter may happen, e.g., when the atoms $A$ and $B$ have different dipole matrix elements and/or different dipole orientations. It is then not difficult to solve Eqs. (35) and (36) analytically. In particular, if the atom $B$ is initially in the lower state, $C_{B}(t=0)=0$, we obtain

$$
\begin{aligned}
& C_{A}=\frac{1}{2 D}\{ {\left[-\frac{1}{2}\left(\Gamma_{A^{*} A}-\Gamma_{B^{*} B}\right)+D\right] e^{D_{+} t / 2} } \\
&\left.+\left[\frac{1}{2}\left(\Gamma_{A^{*} A}-\Gamma_{B^{*} B}\right)+D\right] e^{D_{-} t / 2}\right\}, \\
& C_{B}=\frac{\mathcal{K}_{B^{*} A}}{D}\left(e^{D_{+} t / 2}-e^{D_{-} t / 2}\right),
\end{aligned}
$$

where

$$
\begin{gathered}
D=\left[\frac{1}{4}\left(\Gamma_{A^{*} A}-\Gamma_{B^{*} B}\right)^{2}+4 \mathcal{K}_{A^{*} B} \mathcal{K}_{B^{*} A}\right]^{1 / 2} \\
D_{ \pm}=-\frac{1}{2}\left(\Gamma_{A^{*} A}+\Gamma_{B^{*} B}\right) \pm D
\end{gathered}
$$

When the two atoms are identical and have equivalent positions and dipole orientations with respect to the material surroundings such that the relations

$$
\begin{gathered}
\Gamma_{A^{*} A}=\Gamma_{B^{*} B}, \\
\Gamma_{A^{*} B}=\Gamma_{B^{*} A}, \quad \delta_{A^{*} B}=\delta_{B^{*} A}
\end{gathered}
$$

are valid, we have $\mathcal{K}_{A^{*} B}=\mathcal{K}_{B^{*} A}$, and $\Gamma_{A^{*} B}, \Gamma_{B^{*} A}, \delta_{A^{*} B}$, and $\delta_{B^{*} A}$ are real quantities due to the reciprocity of the Green tensor. Then Eqs. (38) and (39) yield the following expressions for the upper-state occupation probabilities $P_{A(B)}(t)=\left|C_{A(B)}(t)\right|^{2}:$

$$
P_{A(B)}(t)=\frac{1}{2}\left[\cosh \left(\Gamma_{A^{*} B} t\right)+(-) \cos \left(2 \delta_{A^{*} B} t\right)\right] e^{-\Gamma_{B^{*} B} t} .
$$

It is worth noting that Eqs. (38) - 41) [and thus Eq. (44)] are valid for arbitrary dispersing and absorbing material surroundings of the atoms. In particular, substituting in Eqs. (21) and (37) for the Green tensor the vacuum Green tensor, Eq. (44) reduces to that one obtained in Ref. [16]. Accordingly, using the Green tensor for absorbing bulk material, the result in Ref. [6] is recognized.

From Eq. (44) a damped oscillatory excitation exchange between the two atoms is seen. For sufficiently small times, $\Gamma_{B^{*} B} t \ll 1$, and strong resonant dipoledipole coupling, $\left|\delta_{A^{*} B}\right| \gg \Gamma_{B^{*} B}$, the oscillatory behavior dominates. This can typically be observed when the atoms are sufficiently near to each other, but can also be realized for more moderate distances with the interatom coupling being mediated by high- $Q$ mediumassisted field resonances [8]. In the opposite limit of weak resonant dipole-dipole coupling, $P_{A}(t)$ decreases monotonously while $P_{B}(t)$ features one peak which separates the regime of energy transfer from atom $A$ to atom $B$ at early times and the subsequent decay of the excited state of atom $B$.

\section{B. Strong atom-field coupling}

For the sake of transparency, we again consider identical atoms that have equivalent positions and dipole orientations with respect to the material surroundings so 
that Eqs. 42 and (43) hold. Introducing the probability amplitudes

$$
C_{ \pm}(t)=2^{-\frac{1}{2}}\left[C_{A}(t) \pm C_{B}(t)\right] e^{\mp i \delta_{A^{*} B} t}
$$

of the superposition states

$$
| \pm\rangle=2^{-1 / 2}\left(\left|U_{A}\right\rangle \pm\left|U_{B}\right\rangle\right),
$$

from Eqs. (33) we find that the equations for $C_{+}(t)$ and $C_{-}(t)$ decouple,

$$
\dot{C}_{ \pm}(t)=\int_{0}^{t} \mathrm{~d} t^{\prime} \int_{0}^{\infty} \mathrm{d} \omega K_{ \pm}\left(t, t^{\prime} ; \omega\right) e^{\mp i \delta_{A^{*} B}\left(t-t^{\prime}\right)} C_{ \pm}\left(t^{\prime}\right),
$$

where

$$
K_{ \pm}\left(t, t^{\prime} ; \omega\right)=K_{A^{*} A}\left(t, t^{\prime} ; \omega\right) \pm K_{A^{*} B}\left(t, t^{\prime} ; \omega\right) .
$$

Let us restrict our attention to the case when the absolute value of the two-atom term $K_{A^{*} B}\left(t, t^{\prime} ; \omega\right)$ is of the same order of magnitude as the absolute value of the single-atom term $K_{A^{*} A}\left(t, t^{\prime} ; \omega\right)$, so that there is a strong contrast in the magnitude of $K_{+}$and $K_{-}$. Typically true when the atoms are close to each other, this may also take place at interatomic distances much larger than the wavelength, e.g., as in the case of atoms situated at diametrically opposite positions near a microsphere [8]. As a consequence, the strong-coupling regime is applicable to either the state $|+\rangle$ or the state $|-\rangle$, but not to both at the same time. Assuming that the field resonance strongly coupled to the atoms has a Lorentzian shape, with $\omega_{m}$ and $\Delta \omega_{m}$ being the central frequency and the half width at half maximum respectively, we can perform the frequency integral in Eq. (47) in a closed form, on extending it to $\pm \infty$,

$$
\begin{gathered}
\int_{0}^{\infty} \mathrm{d} \omega K_{ \pm}\left(t, t^{\prime} ; \omega\right) \simeq-\frac{\Gamma_{ \pm}}{2 \pi}\left(\Delta \omega_{m}\right)^{2} e^{-i\left(\omega_{m}-\tilde{\omega}_{A}\right)\left(t-t^{\prime}\right)} \\
\times \int_{-\infty}^{+\infty} \mathrm{d} \omega \frac{e^{-i\left(\omega-\omega_{m}\right)\left(t-t^{\prime}\right)}}{\left(\omega-\omega_{m}\right)^{2}+\left(\Delta \omega_{m}\right)^{2}} \\
=-\frac{\Gamma_{ \pm}}{2} \Delta \omega_{m} e^{-i\left(\omega_{m}-\tilde{\omega}_{A}\right)\left(t-t^{\prime}\right)} e^{-\Delta \omega_{m}\left|t-t^{\prime}\right|}
\end{gathered}
$$

where

$$
\Gamma_{ \pm}=\Gamma_{A^{*} A} \pm \Gamma_{A^{*} B}
$$

with $\Gamma_{A^{*} A}$ and $\Gamma_{A^{*} B}$ being defined according to Eq. (21) with $\omega_{m}$ in place of $\tilde{\omega}_{A}\left(\simeq \tilde{\omega}_{B}\right)$. Substituting Eq. (49) into Eq. (47), and differentiating both sides of the resulting equation with regard to time, we arrive at

$$
\begin{array}{r}
\ddot{C}_{ \pm}(t)+\left[i\left(\omega_{m}-\tilde{\omega}_{A} \pm \delta_{A^{*} B}\right)+\Delta \omega_{m}\right] \dot{C}_{ \pm}(t) \\
+\left(\Omega_{ \pm} / 2\right)^{2} C_{ \pm}(t)=0
\end{array}
$$

where

$$
\Omega_{ \pm}=\sqrt{2 \Gamma_{ \pm} \Delta \omega_{m}} .
$$

In particular for exact resonance, i.e., $\omega_{m}=\tilde{\omega}_{A} \mp \delta_{A^{*} B}$, we derive

$$
C_{ \pm}(t)=2^{-\frac{1}{2}} e^{-\Delta \omega_{m} t / 2} \cos \left(\Omega_{ \pm} t / 2\right)
$$

$\left(\Omega_{ \pm} \gg \Delta \omega_{m}\right)$. For the probability amplitudes of the remaining states $|\mp\rangle$, which are weakly coupled to the field, we obtain

$$
C_{\mp}(t)=2^{-\frac{1}{2}} e^{-\Gamma_{\mp} t / 2}
$$

$\left(\Omega_{\mp} \ll \Delta \omega_{m}\right)$. It then follows that

$$
\begin{aligned}
& P_{A(B)}(t)=\frac{1}{4}\left[e^{-\Gamma_{\mp} t}+e^{-\Delta \omega_{m} t} \cos ^{2}\left(\Omega_{ \pm} t / 2\right)\right. \\
& \left.\quad+(-) 2 e^{-\left(\Delta \omega_{m}+\Gamma_{\mp}\right) t / 2} \cos \left(\Omega_{ \pm} t / 2\right) \cos \left(2 \delta_{A^{*} B} t\right)\right] .
\end{aligned}
$$

Here the upper (lower) signs refer to the case where the state $|+\rangle(|-\rangle)$ is strongly coupled to the medium-assisted field.

Even if damping is ignored, $P_{A(B)}(t)$ is not strictly periodic in general, because $\Omega_{ \pm}$and $\delta_{A^{*} B}$ are not necessarily commensurate with each other. Roughly speaking, between three cases of approximately periodic motion may be distinguished.

(i) $4\left|\delta_{A^{*} B}\right| \gg \Omega_{ \pm}, t \ll 2 / \Omega_{ \pm}$:

$$
\begin{aligned}
& P_{A}(t)=\cos ^{2}\left(\delta_{A^{*} B} t\right), \\
& P_{B}(t)=\sin ^{2}\left(\delta_{A^{*} B} t\right) .
\end{aligned}
$$

(ii) $4\left|\delta_{A^{*} B}\right| \simeq \Omega_{ \pm}, t \ll 1 /\left|2 \delta_{A^{*} B}-\Omega_{ \pm} / 2\right|$ :

$$
\begin{aligned}
& P_{A}(t)=\frac{1}{4}\left[1+3 \cos ^{2}\left(\Omega_{ \pm} t / 2\right)\right], \\
& P_{B}(t)=\frac{1}{4}\left[1-\cos ^{2}\left(\Omega_{ \pm} t / 2\right)\right] .
\end{aligned}
$$

(iii) $4\left|\delta_{A^{*} B}\right| \ll \Omega_{ \pm}, t \ll 1 /\left|2 \delta_{A^{*} B}\right|$ :

$$
\begin{aligned}
& P_{A}(t)=\cos ^{4}\left(\Omega_{ \pm} t / 4\right), \\
& P_{B}(t)=\sin ^{4}\left(\Omega_{ \pm} t / 4\right) .
\end{aligned}
$$

From Eqs. (56) - (61) the following time-averaged probabilities $\bar{P}_{A}, \bar{P}_{B}$, and $\bar{P}_{L}=1-\bar{P}_{A}-\bar{P}_{B}$ are obtained, with the respective time integral being taken over one cycle. (i) $\bar{P}_{A}=\bar{P}_{B}=\frac{1}{2}$ and $\bar{P}_{L}=0$. The excitation energy is periodically exchanged between the two atoms through virtual field excitations exclusively. (ii) $\bar{P}_{A}=\frac{5}{8}, \bar{P}_{B}=\frac{1}{8}$, and $\bar{P}_{L}=\frac{2}{8}$. The two exchange channels - one channel through virtual and the other one through real field excitations - compete with each other and destructively interfere, leading to a partial trapping of the excitation energy in atom $A$. Note that this kind of energy transfer suppression cannot be observed, if the interference effect is disregarded as in Ref. [12]. (iii) $\bar{P}_{A}=\bar{P}_{B}=\frac{3}{8}, \bar{P}_{L}=\frac{2}{8}$. This case is typically observed in the long-distance limit, when the interatom energy exchange is dominantly mediated by real field excitations. 


\section{RATE REGIME}

Let us return to the case of weak atom-field coupling. If the resonant dipole-dipole interaction is also weak, then, as already mentioned in Subsection IVA, the energy transfer is one-way; that is, from atom $A$ to atom $B$. Clearly, the efficiency of such a transfer regime is low, because only a small portion of energy is passed on to atom $B$. In this case, a transfer rate $w_{1}$ can be introduced according to

$$
w_{1}=\left.\frac{\mathrm{d} P_{B}(t)}{\mathrm{d} t}\right|_{t_{0}},
$$

where $t_{0}$ is determined from the conditions that

$$
\left.\frac{\mathrm{d}^{2} P_{B}(t)}{\mathrm{d}^{2} t}\right|_{t_{0}}=0,\left.\quad \frac{\mathrm{d} P_{B}(t)}{\mathrm{d} t}\right|_{t_{0}}>0 .
$$

Note that this rate is not much different from that defined as the ratio between the maximum value of $P_{B}(t)$ and the time belonging to it 23. From Eq. (39) [together with Eqs. (40) and (41) for negligibly small $K_{A^{*} B}$ and $K_{B^{*} A}$ therein] it then follows that

$$
\begin{aligned}
w_{1} \simeq & \frac{\left|K_{B^{*} A}\right|^{2}}{D^{2}} e^{D_{-} t_{0}} \\
& \times\left[D_{-}+D_{+} e^{2 D t_{0}}-\left(D_{+}+D_{-}\right) e^{D t_{0}}\right], \\
t_{0} \simeq & \frac{1}{D} \ln \left(\frac { 1 } { 4 D _ { + } ^ { 2 } } \left\{\left(D_{+}+D_{-}\right)^{2}\right.\right. \\
& \left.\left.-2 D\left[\left(D_{+}+D_{-}\right)^{2}+4 D_{+} D_{-}\right]^{1 / 2}\right\}\right),
\end{aligned}
$$

where

$$
\begin{gathered}
D=\frac{1}{2}\left|\Gamma_{A^{*} A}-\Gamma_{B^{*} B}\right|, \\
D_{+(-)}=\left\{\begin{array}{lll}
-\Gamma_{B^{*} B\left(A^{*} A\right)} & \text { if } & \Gamma_{A^{*} A}>\Gamma_{B^{*} B}, \\
-\Gamma_{A^{*} A\left(B^{*} B\right)} & \text { if } & \Gamma_{A^{*} A}<\Gamma_{B^{*} B} .
\end{array}\right.
\end{gathered}
$$

Let us analyze Eq. (64) for three particular cases.

(i) If $\Gamma_{A^{*} A} \gg \Gamma_{B^{*} B}$ is valid, then from Eq. (65) it follows that $\Gamma_{A^{*} A} t_{0}=\ln 4$, and Eq. (64) reduces to

$$
w_{1}=\left|\mathcal{K}_{B^{*} A}\right|^{2} / \Gamma_{A * A} .
$$

(ii) For $\Gamma_{A^{*} A}=\Gamma_{B^{*} B}$ the relation $\Gamma_{B^{*} B} t_{0}=2-\sqrt{2}$ holds. Thus, Eq. (64) reads as

$$
w_{1} \simeq\left|\mathcal{K}_{B^{*} A}\right|^{2} 2(\sqrt{2}-1) e^{-(2-\sqrt{2})} / \Gamma_{B^{*} B}
$$

(iii) In the case where $\Gamma_{B^{*} B} \gg \Gamma_{A^{*} A}$ is valid, one finds $\Gamma_{B^{*} B} t_{0}=\ln 4$, so that Eq. (64) reduces to

$$
w_{1}=\left|\mathcal{K}_{B^{*} A}\right|^{2} / \Gamma_{B^{*} B}
$$

A typical example of the temporal evolution of $P_{B}(t)$ for weak resonant dipole-dipole interaction is plotted in Fig. 11 for the case (ii). The rate regime with linear time dependence is seen to be established after some short time interval where a $t^{2}$-dependence is observed.

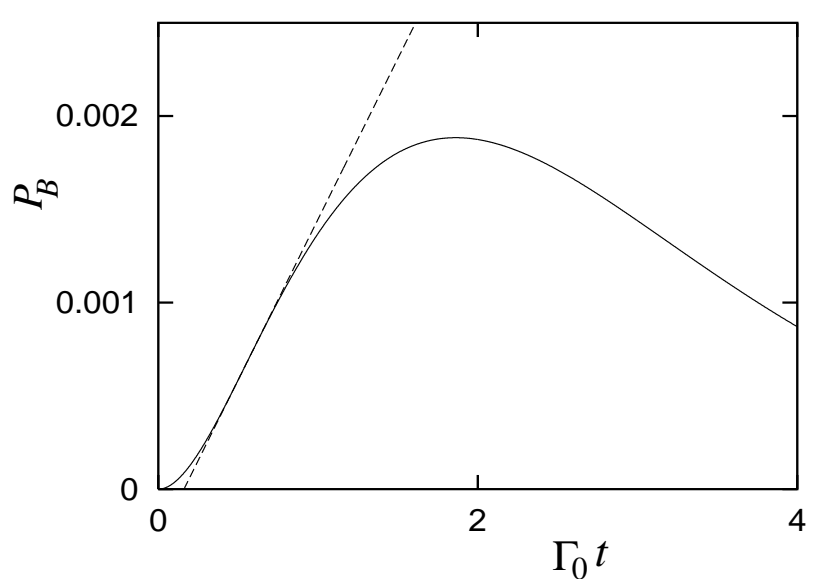

FIG. 1. The probability of finding atom $B$ in the upper state as a function of time for two atoms situated at diametrically opposite positions outside a dielectric microsphere. The parameters have been chosen such that $\Gamma_{A^{*} A}=\Gamma_{B^{*} B}=1.07 \Gamma_{0}, \Gamma_{A^{*} B}=0.04 \Gamma_{0}$, and $\delta_{A^{*} B}=0.06 \Gamma_{0}$, $\Gamma_{0}$ being the single-atom decay rate in free space. The solid curve is exact, in accordance with Eq. (44), and the dashed curve is $P_{B}(t) \simeq P_{B}\left(t_{0}\right)+w_{1}\left(t-t_{0}\right)$.

Another possible way for determining an energy transfer rate is to infer it directly from the equations of motion. From Eq. (24) [or Eqs. (35) and (36)] we find that

$$
\begin{gathered}
\dot{\varrho}_{B B}=-\Gamma_{B^{*} B} \varrho_{B B}+\left(\mathcal{K}_{B^{*} A} \varrho_{A B}+\text { c.c. }\right), \\
\dot{\varrho}_{A B}=-\frac{1}{2}\left(\Gamma_{A^{*} A}+\Gamma_{B^{*} B}\right) \varrho_{A B} \\
\quad+\mathcal{K}_{A^{*} B} \varrho_{B B}+\mathcal{K}_{B^{*} A}^{*} \varrho_{A A}
\end{gathered}
$$

where the notation

$$
\varrho_{A A^{\prime}}=\left\langle U_{A}|\hat{\varrho}| U_{A^{\prime}}\right\rangle=C_{A} C_{A^{\prime}}^{*}
$$

is used $\left(\tilde{\omega}_{A}=\tilde{\omega}_{A^{\prime}}\right)$. In the rate regime $\varrho_{B B}\left(=P_{B}\right)$ varies (approximately) linearly with time. This may happen for times where $\varrho_{A B}$ may be regarded as being (quasi-)stationary. By setting $\dot{\varrho}_{A B}=0$ in Eq. (72) and substituting the resulting expression for $\varrho_{A B}$ into Eq. (71), we obtain

$$
\begin{aligned}
\dot{\varrho}_{B B}=-\left[\Gamma_{B^{*} B}-\right. & \left.\left(\frac{2 \mathcal{K}_{A^{*} B} \mathcal{K}_{B^{*} A}}{\Gamma_{A A^{*}}+\Gamma_{B^{*} B}}+\text { c.c. }\right)\right] \varrho_{B B} \\
& +\frac{4\left|\mathcal{K}_{B^{*} A}\right|^{2}}{\Gamma_{A^{*} A}+\Gamma_{B^{*} B}} \varrho_{A A}
\end{aligned}
$$


In the rate regime, the small first term in Eq. (74) may be neglected, and in the second term $\varrho_{A A}$ may be replaced with an appropriately chosen $P_{A}^{(0)}=\varrho_{A A}^{(0)}$. The energy transfer rate obtained in this way is then given by

$$
w_{2}=\frac{4\left|\mathcal{K}_{B^{*} A}\right|^{2}}{\Gamma_{A^{*} A}+\Gamma_{B^{*} B}} P_{A}^{(0)}
$$

The interest in energy transfer processes has stemmed from their key role in a wide variety of both biological and nonbiological systems, where the species participating in the energy transfer are typically (not necessarily identical) molecules with dense manifolds of vibronic states. Commonly, a rate regime is considered and the transfer rate is calculated by means of Fermi's golden rule in second-order perturbation theory with regard to the molecule-field interaction,

$$
w=\sum_{f, i} p_{i} w_{f i}
$$

where $w_{f i}$ is proportional to the absolute square of the second-order interaction matrix element and the energy conserving $\delta$-function $\delta\left(\omega_{f}-\omega_{i}\right)$, and the sum runs over the continuum of initial $(i)$ and/or final $(f)$ states. In particular, short-distance energy transfer, where the intermolecular coupling is essentially static, has been well known as Förster transfer [24]. Later on energy transfer over arbitrary distances has been considered (see, e.g., Refs. [6, 14 and references therein). The energy transfer rate in the presence of dispersing and absorbing material surroundings has been calculated in Ref. 14 on the basis of a Hamiltonian of the type given in Eq. (2).

For two two-level atoms the single-transition probability per unit time $w_{i f}$ corresponds to $\dot{P}_{B}$. Starting from the effective Hamiltonian (27) and evaluating Eq. (33) (for $C_{B}$ ) in first-order perturbation theory, we derive, on making the standard long-time assumption,

$$
\dot{P}_{B}=2 \pi\left|\mathcal{K}_{B^{*} A}\right|^{2} \delta\left(\tilde{\omega}_{A}-\tilde{\omega}_{B}\right)
$$

(Appendix Q), which is fully consistent with the result, obtained in the standard second-order perturbation theory on the basis of the original (fundamental) Hamiltonian (2) 14. The only new feature is in the current treatment the medium induced atomic frequency shifts are taken into account. Note that the transition matrix element is determined by the full Green tensor.

For establishing a rate regime, it is necessary that, according to Eq. (76), a continuum of initial and/or final states is involved in the transition. In the two-atom problem at hand, the continua of states are obviously provided by the atomic level broadening due to the spontaneous decay. Let $\nu_{A}$ and $\nu_{B}$ be the frequencies of the continua associated with atom $A$ and $B$ respectively and $\xi_{A}\left(\nu_{A}\right)$ and $\xi_{B}\left(\nu_{B}\right)$ the densities of the respective continua. From Eqs. (76) and $(77)$ the interatomic energy transfer rate is then expected to be

$$
\begin{aligned}
w=2 \pi \int & \mathrm{d} \nu_{A} \int \mathrm{d} \nu_{B} \xi_{A}\left(\nu_{A}\right) \xi_{B}\left(\nu_{B}\right) \\
& \times p_{A}\left(\nu_{A}\right)\left|\mathcal{K}_{B^{*} A}\right|^{2} \delta\left(\nu_{A}-\nu_{B}\right) .
\end{aligned}
$$

Assuming Lorentzian line shapes

$$
\xi_{A^{\prime}}\left(\nu_{A^{\prime}}\right)=\frac{1}{\pi} \frac{\Gamma_{A^{\prime *} A^{\prime}} / 2}{\left(\nu_{A^{\prime}}-\tilde{\omega}_{A^{\prime}}\right)^{2}+\left(\Gamma_{A^{\prime *} A^{\prime}} / 2\right)^{2}} \quad\left(A^{\prime}=A, B\right),
$$

and making the assumption that $p_{A}\left(\nu_{A}\right)$ and $\left|\mathcal{K}_{B^{*} A}\right|^{2}$ as functions of frequency slowly vary over the lines, we may perform the integrals in Eq. (78) to obtain

$$
w=\frac{4\left|\mathcal{K}_{B^{*} A}\right|^{2}}{\Gamma_{A^{*} A}+\Gamma_{B^{*} B}} p_{A}\left(\tilde{\omega}_{A}\right)
$$

which exactly agrees with the rate $w_{2}$ given by Eq. (75), if the assumption is made that

$$
p_{A}\left(\tilde{\omega}_{A}\right) \simeq P_{A}^{(0)}
$$

Finally, let us identify $p_{A}\left(\tilde{\omega}_{A}\right)$ in Eq. 80 ) for the perturbative rate $w$ with $P_{A}\left(t_{0}\right)$ [Eq. (55) together with Eq. (65)] and compare the result with the nonperturbative rate $w_{1}$ calculated from the exact temporal evolution of $P_{B}(t)$ [Eq. (64) together with Eq. (65)]. Noting that $P_{A}\left(t_{0}\right) \simeq \exp \left(-\Gamma_{A^{*} A} t_{0}\right)$, we find for the three cases considered in Eqs. 68) - (70) the following results.

(i) $\Gamma_{A^{*} A} \gg \Gamma_{B^{*} B}$ :

$$
P_{A}\left(t_{0}\right) \simeq \frac{1}{4}, \quad \frac{w_{1}}{w} \simeq 1
$$

(ii) $\Gamma_{A^{*} A}=\Gamma_{B^{*} B}$ :

$$
P_{A}\left(t_{0}\right) \simeq e^{-(2-\sqrt{2})}, \quad \frac{w_{1}}{w} \simeq \sqrt{2}-1 \simeq 0.41 .
$$

(iii) $\Gamma_{B^{*} B} \gg \Gamma_{A^{*} A}$ :

$$
P_{A}\left(t_{0}\right) \simeq 1, \quad \frac{w_{1}}{w} \simeq \frac{1}{4}
$$

Agreement between $w_{1}$ and $w$ is only observed in the first case, where $\Gamma_{B^{*} B}$ is sufficiently small. With increasing value of $\Gamma_{B * B}$ an increasing discrepancy between $w_{1}$ and $w$ is observed.

The reason can be seen in the fact that there are two processes which simultaneously drive atom $B$ : the energy transfer from $A$ to $B$ and the spontaneous decay. Hence, $w_{1}$ is actually the rate of both processes combined, not the "naked" rate of energy transfer. This explains why $w_{1}$ is typically smaller than $w$ and why the discrepancy between them becomes more substantial for enhanced spontaneous decay of atom $B$. Note that the role of the spontaneous decay of atom $B$ is twofold. On 
the one hand, it takes away the population of the upper state of atom $B$, thus diminishes $w_{1}$ relative to $w$. On the other hand, it leads to an atomic level broadening which must carefully be taken into account for a proper evaluation of the rate $w$ according to Eq. (78). To roughly compensate for the first effect, the ratio $w_{1} / w$ may be multiplied by $\exp \left(\Gamma_{B^{*} B} t_{0}\right)$. The values of the so corrected ratio are then $\simeq 1$ for the cases (i) and (iii), and $\simeq 0.74$ for the case (ii).

\section{POWER SPECTRUM}

Let us finally address the question of the influence of the resonant dipole-dipole interaction on the power spectrum of light emitted by two identical atoms that initially share a single excitation. As is well known, the (physical) spectrum can be obtained by a Fourier transformation of the two-time correlation function of the electric-field strength in normal order (see, e.g., 25)

$$
\begin{array}{rl}
S\left(\mathbf{r}, \omega_{\mathrm{S}}, T\right)=\int_{0}^{T} & \mathrm{~d} t_{2} \int_{0}^{T} \mathrm{~d} t_{1}\left[e^{-i \omega_{\mathrm{S}}\left(t_{2}-t_{1}\right)}\right. \\
& \left.\times\left\langle\hat{\mathbf{E}}^{(-)}\left(\mathbf{r}, t_{2}\right) \hat{\mathbf{E}}^{(+)}\left(\mathbf{r}, t_{1}\right)\right\rangle\right],
\end{array}
$$

where $\omega_{\mathrm{S}}$ is the setting frequency of the (ideal) spectral apparatus, $T$ is the operating-time interval of the detector, and

$$
\begin{gathered}
\hat{\mathbf{E}}^{(+)}(\mathbf{r})=\int_{0}^{\infty} \mathrm{d} \omega \underline{\hat{\mathbf{E}}}(\mathbf{r}, \omega), \\
\hat{\mathbf{E}}^{(-)}(\mathbf{r})=\left[\hat{\mathbf{E}}^{(+)}(\mathbf{r})\right]^{\dagger}
\end{gathered}
$$

\section{A. Weak atom-field coupling}

For weak atom-field coupling we derive, on basing on Eqs. (38), (39), 42, and (43),

$$
\begin{aligned}
S\left(\mathbf{r}, \omega_{S}, T \rightarrow \infty\right)= & \frac{1}{4} \mid \frac{\mathbf{F}_{A}+\mathbf{F}_{B}}{\Delta \omega_{S}+\delta_{A^{*} B}+i \Gamma_{+} / 2} \\
& +\left.\frac{\mathbf{F}_{A}-\mathbf{F}_{B}}{\Delta \omega_{S}-\delta_{A^{*} B}+i \Gamma_{-} / 2}\right|^{2}
\end{aligned}
$$

where

$$
\Delta \omega_{S}=\omega_{S}-\tilde{\omega}_{A}
$$

and $\left(A^{\prime}=A, B\right)$

$$
\mathbf{F}_{A^{\prime}}=\frac{\tilde{\omega}_{A^{\prime}}^{2}}{\pi \epsilon_{0} c^{2}} \int_{0}^{\prime \infty} \mathrm{d} \omega \operatorname{Im} \boldsymbol{G}\left(\mathbf{r}, \mathbf{r}_{\mathrm{A}^{\prime}}, \omega\right) \mathbf{d}_{A^{\prime}} \zeta\left(\tilde{\omega}_{A^{\prime}}-\omega\right)
$$

(Appendix D). Note that replacing $\zeta\left(\tilde{\omega}_{A^{\prime}}-\omega\right)$ with $\pi \delta\left(\tilde{\omega}_{A^{\prime}}-\omega\right)$ would be too rough here. Equation (88) reveals that the emitted light is spectrally split into two asymmetric lines at $\tilde{\omega}_{A} \mp \delta_{A^{*} B}$, with $\Gamma_{ \pm}$and $\left|\mathbf{F} A \pm \mathbf{F}_{B}\right|^{2}$ being the widths and weights respectively. The line separation is seen to be twice the dipole-dipole coupling parameter. A system of two two-level atoms, one of them initially excited, is obviously equivalent to a three-level system with two upper dressed states $| \pm\rangle$. The doublet structure of the emitted-light spectrum can be easily understood as a result of the transitions of the dressed states to the ground state. For the case of the atoms being in vacuum, similar results were found not only for the spontaneous emission [16] but also for the resonance fluorescence (see, e.g., Ref. 26 and references therein).

As can be seen from Eq. (88), an experimental observation of doublet structure of the emitted light requires a delicate balancing act. The interatomic distance should not be too large to provide a reasonable level splitting, but it should not be too small to avoid $\left|\mathbf{F}_{A}\right|=\left|\mathbf{F}_{B}\right|$, i.e., quenching of one of the two lines. It is worth noting that the presence of macroscopic bodies may facilitate the detection of the doublet, because it offers the possibility of realizing strong resonant dipole-dipole interaction even for interatomic distances much larger than the wavelength.

Another interesting feature is that, according to Eq. (50), either $\Gamma_{+}$or $\Gamma_{-}$, can be much smaller than $\Gamma_{A^{*} A}\left(=\Gamma_{B^{*} B}\right)$. That is, the resonant dipole-dipole interaction can give rise to an ultranarrow spectral line, albeit each time at the expense of the other line of the pair. If, e.g., a single atom is placed sufficiently near a microsphere, its spontaneous decay may be suppressed, with the emission line being accordingly narrowed. Compared to the emission line of a single atom, one line of the doublet observed for two atoms being present may be further narrowed by several orders of magnitude [8].

\section{B. Strong atom-field coupling}

For strong atom-field coupling, Eq. (88) changes to, on basing on Eqs. (53) and (54),

$$
\begin{gathered}
S\left(\mathbf{r}, \omega_{S}, T \rightarrow \infty\right)=\frac{1}{4} \mid\left(\mathbf{W}_{A} \pm \mathbf{W}_{B}\right) \\
\times\left(\frac{1}{\Delta \omega_{S} \pm \delta_{A^{*} B}+\Omega_{ \pm} / 2+i \Delta \omega_{m} / 2}\right. \\
\left.-\frac{1}{\Delta \omega_{S} \pm \delta_{A^{*} B}-\Omega_{ \pm} / 2+i \Delta \omega_{m} / 2}\right) \\
+\left.\frac{i\left(\mathbf{F}_{A} \mp \mathbf{F}_{B}\right)}{\Delta \omega_{S} \mp \delta_{A^{*} B}+i \Gamma_{\mp} / 2}\right|^{2}
\end{gathered}
$$

where $\left(A^{\prime}=A, B\right)$ 


$$
\mathbf{W}_{A^{\prime}}=\frac{\omega_{m}^{2} \Delta \omega_{m}}{\epsilon_{0} c^{2} \Omega_{ \pm}} \operatorname{Im} \boldsymbol{G}\left(\mathbf{r}, \mathbf{r}_{\mathrm{A}^{\prime}}, \omega_{m}\right) \mathbf{d}_{A^{\prime}}
$$

(Appendix D). Here the upper (lower) signs again refer to the case where the state $|+\rangle(|-\rangle)$ is strongly coupled to the medium-assisted field. Eq. (91) reveals that due to the strong atom-field coupling, the doublet observed for weak atom-field coupling may become a triplet, with one of the lines of the doublet being split into two lines. These lines separated by $\Omega_{ \pm}$have equal widths (which are solely determined by the width of the medium-assisted field resonance) and equal weights. Note that their width and weight are different from those of the third line, which is closely related to a line of the doublet observed for weak atom-field coupling. From Eq. (91) it is also seen that, depending on the point of observation, this line or the strong-coupling-assisted doublet can be suppressed due to the interference effects.

\section{SUMMARY AND CONCLUDING REMARKS}

We have studied the interaction of two-level atoms with the electromagnetic field in the presence of dispersing and absorbing material surroundings described by a spatially varying permittivity that is a complex function of frequency. Starting from the exact multipolar Hamiltonian (in electric-dipole approximation), we have derived reduced density-matrix equations of motion that describe both the on-resonant interaction of the atoms with the medium-assisted electromagnetic field and the resonant dipole-dipole interaction of the atoms with each other via off-resonant atom-field interaction. The equations of motion apply to atoms in the presence of arbitrarily configured, dispersing and absorbing media and are not restricted to the rotating-wave approximation. Whereas the resonant dipole-dipole interaction is essentially controlled by the real part of the medium-assisted Green tensor, the relevant quantity for the resonant atom-field interaction is the imaginary part of the Green tensor. Both together determine the mutual interaction of the atoms.

We have shown that when the differences between the atomic transition frequencies are small compared to the frequency scale of variation of the medium-assisted Green tensor, then the equations of motion can be derived from an effective Hamiltonian. All coupling parameters are again expressed in terms of the medium-assisted Green tensor. The use of the effective Hamiltonian may substantially simplify the calculations in cases where identical atoms are considered. Applying the theory to the two-atom case by assuming that initially the mediumassisted electromagnetic field is in the ground state and one of the atoms is in the upper state, we have considered the resonant energy transfer between the atoms and the spectrum of the emitted radiation.

There are two energy-transfer channels in general: one channel through resonant dipole-dipole interaction medi- ated by virtual-photon creation and destruction and the other one through emission and absorption of real photons. In particular for strong atom-field interaction, the (over a period averaged) energy transfer can be inhibited due to destructive interference of the two available transfer channels. When the dipole-dipole interaction is weak, the energy transfer from the donor atom to the acceptor atom becomes irreversible, and a transfer rate can be inferred from the (exact) temporal evolution of the excited-state population of the acceptor atom. Such a rate regime is commonly described by a transition probability per unit time, such as the Förster transfer rate, which is calculated by means of Fermi's golden rule in second order perturbation theory with respect to the fundamental Hamiltonian. We have shown that the two methods essentially lead to the same expression for the transfer rate. In any case it is proportional to the absolute square of the medium-assisted (full) Green tensor. In contrast to molecules, where the necessary continuum of initial/final states is typically given by the vibronic states, in the case of atoms this continuum is essentially built up during the process of spontaneous decay.

The results show that both the resonant energy transfer and the doublet spectrum of the emitted light observed for weak atom-field coupling or the triplet spectrum observed for strong atom-field coupling can be controlled by the presence of macroscopic bodies. Clearly, the present analysis has left a number of open questions, on which future work will concentrate. In particular, the problem of energy exchange between atoms whose transition frequencies must be regarded as being different with regard to the variation of the medium-assisted Green tensor, needs special emphasis.

\section{ACKNOWLEDGMENTS}

This work was supported by the Deutsche Forschungsgemeinschaft.

\section{APPENDIX A: DERIVATION OF Eqs. (8) and (19)}

In order to perform the second term in Eq. (5),

$$
\begin{aligned}
\hat{F}^{\prime \prime}(t)=\frac{i}{\hbar} \sum_{A} & \int_{0}^{\prime \prime \infty} \mathrm{d} \omega\left\{\left[\hat{O}(t), \hat{\mathbf{d}}_{A}(t)\right] \underline{\hat{\mathbf{E}}}\left(\mathbf{r}_{A}, \omega, t\right)\right. \\
& \left.+\underline{\hat{\mathbf{E}}}^{\dagger}\left(\mathbf{r}_{A}, \omega, t\right)\left[\hat{O}(t), \hat{\mathbf{d}}_{A}(t)\right]\right\},
\end{aligned}
$$

we formally integrate Eq. (7) to obtain

$$
\hat{\mathbf{f}}(\mathbf{r}, \omega, t)=\hat{\mathbf{f}}_{\text {free }}(\mathbf{r}, \omega, t)+\frac{\omega^{2}}{c^{2}} \sqrt{\frac{\varepsilon_{\mathrm{I}}(\mathbf{r}, \omega)}{\hbar \pi \varepsilon_{0}}}
$$




$$
\times \sum_{A} \int_{0}^{t} \mathrm{~d} t^{\prime} \hat{\mathbf{d}}_{A}\left(t^{\prime}\right) \boldsymbol{G}\left(\mathbf{r}_{A}, \mathbf{r}, \omega\right) e^{-i \omega\left(t-t^{\prime}\right)},
$$

where $\hat{\mathbf{f}}_{\text {free }}(\mathbf{r}, \omega, t)$ evolves freely. Inserting Eq. A2 into Eq. (何), we derive

$$
\begin{aligned}
& \underline{\hat{\mathbf{E}}}(\mathbf{r}, \omega, t)=\underline{\hat{\mathbf{E}}}_{\mathrm{free}}(\mathbf{r}, \omega, t) \\
& +\frac{i}{\pi \varepsilon_{0}} \frac{\omega^{2}}{c^{2}} \sum_{A} \int_{0}^{t} \mathrm{~d} t^{\prime} e^{-i \omega\left(t-t^{\prime}\right)} \operatorname{Im} \boldsymbol{G}\left(\mathbf{r}, \mathbf{r}_{A}, \omega\right) \hat{\mathbf{d}}_{A}\left(t^{\prime}\right),
\end{aligned}
$$

where $\underline{\mathbf{E}}_{\text {free }}(\mathbf{r}, \omega, t)$ is defined according to Eq. (位) with $\hat{\mathbf{f}}_{\text {free }}(\mathbf{r}, \omega, t)$ in place of $\hat{\mathbf{f}}(\mathbf{r}, \omega, t)$.

Let us first restrict our attention to the single-atom case. Introducing slowly varying atomic operator

$$
\hat{\tilde{\sigma}}_{A}(t)=\hat{\sigma}_{A}(t) e^{i \omega_{A} t},
$$

we may rewrite Eq. (3) as

$$
\hat{\mathbf{d}}_{A}(t)=\mathbf{d}_{A} \hat{\tilde{\sigma}}_{A}(t) e^{-i \omega_{A} t}+\mathbf{d}_{A}^{*} \hat{\tilde{\sigma}}_{A}^{\dagger}(t) e^{i \omega_{A} t} .
$$

Inserting Eqs. (A3) and (A5) into Eq. (A1), we obtain

$$
\begin{gathered}
\hat{F}_{A}^{\prime \prime}(t)=\hat{F}_{A \text { free }}^{\prime \prime}-\frac{1}{\hbar \pi \varepsilon_{0}} \int_{0}^{t} \mathrm{~d} t^{\prime} \int_{0}^{\prime \prime \infty} \mathrm{d} \omega \frac{\omega^{2}}{c^{2}}\left\{\left[\hat{O}(t), \hat{\mathbf{d}}_{A}(t)\right]\right. \\
\times \operatorname{Im} \boldsymbol{G}\left(\mathbf{r}_{A}, \mathbf{r}_{A}, \omega\right)\left[\mathbf{d}_{A} \hat{\tilde{\sigma}}_{A}\left(t^{\prime}\right) e^{-i\left(\omega-\omega_{A}\right)\left(t-t^{\prime}\right)} e^{-i \omega_{A} t}\right. \\
\left.\quad+\mathbf{d}_{A}^{*} \hat{\tilde{\sigma}}_{A}^{\dagger}\left(t^{\prime}\right) e^{-i\left(\omega+\omega_{A}\right)\left(t-t^{\prime}\right)} e^{i \omega_{A} t}\right] \\
-\operatorname{Im} \boldsymbol{G}\left(\mathbf{r}_{A}, \mathbf{r}_{A}, \omega\right)\left[\mathbf{d}_{A} \hat{\tilde{\sigma}}_{A}\left(t^{\prime}\right) e^{i\left(\omega+\omega_{A}\right)\left(t-t^{\prime}\right)} e^{-i \omega_{A} t}\right. \\
\left.\left.+\mathbf{d}_{A}^{*} \hat{\tilde{\sigma}}_{A}^{\dagger}\left(t^{\prime}\right) e^{i\left(\omega-\omega_{A}\right)\left(t-t^{\prime}\right)} e^{i \omega_{A} t}\right]\left[\hat{O}(t), \hat{\mathbf{d}}_{A}(t)\right]\right\}, \quad \mathrm{A}(6)
\end{gathered}
$$

where

$$
\begin{aligned}
\hat{F}_{A \text { free }}^{\prime \prime}(t)=\frac{i}{\hbar} & \int_{0}^{\prime \prime \infty} \mathrm{d} \omega\left\{\left[\hat{O}(t), \hat{\mathbf{d}}_{A}(t)\right] \underline{\mathbf{E}}_{\text {free }}\left(\mathbf{r}_{A}, \omega, t\right)\right. \\
& \left.+\underline{\mathbf{E}}_{\text {free }}^{\dagger}\left(\mathbf{r}_{A}, \omega, t\right)\left[\hat{O}(t), \hat{\mathbf{d}}_{A}(t)\right]\right\} .
\end{aligned}
$$

Confining ourselves to resolving times large compared with $1 /\left(\omega-\omega_{A}\right)$, we may regard the exponential functions in Eq. (A6) as being rapidly varying with time. Recall that range of integration only covers off-resonant frequencies. In the time integrals, we may then replace the slowly varying operator $\hat{\tilde{\sigma}}_{A}\left(t^{\prime}\right)$ by $\hat{\tilde{\sigma}}_{A}(t)$ (Markov approximation). In the spirit of the coarse-grained time averaging mentioned, we may further make the replacement

$$
\int_{0}^{t} \mathrm{~d} t^{\prime} e^{-i\left(\omega-\omega_{A}\right)\left(t-t^{\prime}\right)} \rightarrow \zeta\left(\omega_{A}-\omega\right)
$$

$[\zeta(x)=\pi \delta(x)+i \mathcal{P} / x]$ and drop the terms where $\hat{\tilde{\sigma}}_{A}$ or $\hat{\tilde{\sigma}}_{A}^{\dagger}$ appears twice. In this way, Eq. (A6) approximately reads as

$$
\begin{aligned}
& \hat{F}_{A}^{\prime \prime}(t) \simeq \hat{F}_{A \text { free }}^{\prime \prime}(t) \\
&-\frac{1}{\hbar \pi \varepsilon_{0}} \int_{0}^{\prime \prime \infty} \mathrm{d} \omega \frac{\omega^{2}}{c^{2}} \mathbf{d}_{A}^{*} \operatorname{Im} \boldsymbol{G}\left(\mathbf{r}_{A}, \mathbf{r}_{A}, \omega\right) \mathbf{d}_{A} \\
& \times\left\{\zeta\left[-\left(\omega+\omega_{A}\right)\right]\left[\hat{O}(t), \hat{\sigma}_{A}(t)\right] \hat{\sigma}_{A}^{\dagger}(t)\right. \\
& \quad+\zeta\left(\omega_{A}-\omega\right)\left[\hat{O}(t), \hat{\sigma}_{A}^{\dagger}(t)\right] \hat{\sigma}_{A}(t) \\
&-\zeta\left(\omega+\omega_{A}\right) \hat{\sigma}_{A}(t)\left[\hat{O}(t), \hat{\sigma}_{A}^{\dagger}(t)\right] \\
&\left.\quad-\zeta\left(\omega-\omega_{A}\right) \hat{\sigma}_{A}^{\dagger}(t)\left[\hat{O}(t), \hat{\sigma}_{A}(t)\right]\right\} .
\end{aligned}
$$

Substituting Eq. (A9) into Eq. (5) and taking into account that, because of $\omega \pm \omega_{A} \neq 0$, the $\delta$-function parts of the $\zeta$-functions do not contribute to the frequency integrals, we make the approximation

$$
\zeta\left(\omega \pm \omega_{A}\right) \rightarrow i \mathcal{P} /\left(\omega \pm \omega_{A}\right)
$$

and arrive at

$$
\dot{\hat{O}}=-\frac{i}{\hbar}\left[\hat{O}, \hat{\tilde{H}}_{A \mathrm{~S}}\right]+\hat{F}_{A \text { free }}^{\prime \prime} .
$$

Here, $\hat{\tilde{H}}_{A \mathrm{~S}}$ is the single-atom system Hamiltonian defined according to Eq. (6), with the "naked" transition frequency $\omega_{A}$ being replaced by the shifted frequency $\tilde{\omega}_{A}=\omega_{A}-\delta_{A^{*} A}$, where the frequency shift $\delta_{A^{*} A}$ is given by Eq. (11) together with Eq. (12).

Let us turn to the multi-atom case. Substituting the formal solution (A3) into Eq. (A1), we now obtain a double-sum over $A$ and $A^{\prime}$,

$$
\hat{F}^{\prime \prime}(t)=\sum_{A} \hat{F}_{A}^{\prime \prime}(t)+\sum_{A, A^{\prime}}^{\prime} \hat{F}_{A A^{\prime}}^{\prime \prime}(t) .
$$

The terms $\hat{F}_{A}^{\prime \prime}(t)$ are treated as described above to give the shifted transition frequency for each atom. In the terms $\hat{F}_{A A^{\prime}}^{\prime \prime}(t)\left(A \neq A^{\prime}\right)$ we then take into account the level shifts and introduce the slowly varying atomic operators according to

$$
\hat{\tilde{\sigma}}_{A}(t)=\hat{\sigma}_{A}(t) e^{i \tilde{\omega}_{A} t} .
$$

By repeating for the terms $\hat{F}_{A A^{\prime}}^{\prime \prime}(t)$ the same procedure as in the single-atom case, it is not difficult to prove that [in place of Eq. (A9)] the result is

$$
\begin{array}{r}
\hat{F}_{A A^{\prime}}^{\prime \prime}(t) \simeq-\frac{1}{\hbar \pi \varepsilon_{0}} \int_{0}^{\prime \prime \infty} \mathrm{d} \omega \frac{\omega^{2}}{c^{2}}\left\{\mathbf{d}_{A} \operatorname{Im} \boldsymbol{G}\left(\mathbf{r}_{A}, \mathbf{r}_{A^{\prime}}, \omega\right) \mathbf{d}_{A^{\prime}}^{*}\right. \\
\times \zeta\left[-\left(\omega+\tilde{\omega}_{A^{\prime}}\right)\right]\left[\hat{O}(t), \hat{\sigma}_{A}(t)\right] \hat{\sigma}_{A^{\prime}}^{\dagger}(t) \\
+\mathbf{d}_{A}^{*} \operatorname{Im} \boldsymbol{G}\left(\mathbf{r}_{A}, \mathbf{r}_{A^{\prime}}, \omega\right) \mathbf{d}_{A^{\prime}} \zeta\left(\tilde{\omega}_{A^{\prime}}-\omega\right)\left[\hat{O}(t), \hat{\sigma}_{A}^{\dagger}(t)\right] \hat{\sigma}_{A^{\prime}}(t) \\
-\mathbf{d}_{A}^{*} \operatorname{Im} \boldsymbol{G}\left(\mathbf{r}_{A}, \mathbf{r}_{A^{\prime}}, \omega\right) \mathbf{d}_{A^{\prime}} \zeta\left(\omega+\tilde{\omega}_{A^{\prime}}\right) \hat{\sigma}_{A^{\prime}}(t)\left[\hat{O}(t), \hat{\sigma}_{A}^{\dagger}(t)\right] \\
\left.-\mathbf{d}_{A} \operatorname{Im} \boldsymbol{G}\left(\mathbf{r}_{A}, \mathbf{r}_{A^{\prime}}, \omega\right) \mathbf{d}_{A^{\prime}}^{*} \zeta\left(\omega-\tilde{\omega}_{A^{\prime}}\right) \hat{\sigma}_{A^{\prime}}^{\dagger}(t)\left[\hat{O}(t), \hat{\sigma}_{A}(t)\right]\right\} .
\end{array}
$$


In Eq. A14 we now make the approximation

$$
\zeta\left(\omega \pm \tilde{\omega}_{A^{\prime}}\right) \rightarrow i \mathcal{P} /\left(\omega \pm \tilde{\omega}_{A^{\prime}}\right),
$$

[cf. Eq. (A10)] and insert the resulting expression for $\hat{F}_{A A^{\prime}}^{\prime \prime}(t)$ into Eq. (A12). We eventually combine Eqs. (5) and (A12) and obtain, on again dropping off-resonant terms,

$$
\begin{aligned}
& \dot{\hat{O}}=-\frac{i}{\hbar}\left[\hat{O}, \hat{\tilde{H}}_{\mathrm{S}}\right]+\hat{F}_{\text {free }}^{\prime \prime} \\
&+i \sum_{A, A^{\prime}}^{\prime}\left\{\delta_{A^{*} A^{\prime}}^{-}\left[\hat{O}, \hat{\sigma}_{A}^{\dagger}\right] \hat{\sigma}_{A^{\prime}}+\delta_{A A^{\prime *}}^{+}\left[\hat{O}, \hat{\sigma}_{A}\right] \hat{\sigma}_{A^{\prime}}^{\dagger}\right. \\
&\left.\quad+\delta_{A A^{\prime *}}^{-} \hat{\sigma}_{A^{\prime}}^{\dagger}\left[\hat{O}, \hat{\sigma}_{A}\right]+\delta_{A^{*} A^{\prime}}^{+} \hat{\sigma}_{A^{\prime}}\left[\hat{O}, \hat{\sigma}_{A}^{\dagger}\right]\right\}
\end{aligned}
$$

where

$$
\hat{F}_{\text {free }}^{\prime \prime}=\sum_{A} \hat{F}_{A \text { free }}^{\prime \prime}
$$

and $\delta_{A A^{\prime}}$ is given by Eq. (13) together with Eq. (14). From Eq. (A7) it is seen that when the off-resonant free field is in the vacuum state, then

$$
\left\langle\hat{F}_{\text {free }}^{\prime \prime}\right\rangle=0
$$

is valid, and the expectation value of the operator equation (A16) just yields Eq. (8).

To prove Eq. (19), we use the cyclic properties of the trace and rewrite Eq. (8) as

$$
\begin{aligned}
\frac{\mathrm{d}}{\mathrm{d} t}\langle\hat{O}\rangle= & \operatorname{Tr}\left(\left\{-\frac{i}{\hbar}\left[\hat{\tilde{H}}_{\mathrm{S}}, \hat{\varrho}\right]\right.\right. \\
+i \sum_{A, A^{\prime}}^{\prime} & {\left[\delta_{A^{*} A^{\prime}}^{-}\left(\hat{\sigma}_{A}^{\dagger} \hat{\sigma}_{A^{\prime}} \hat{\varrho}-\hat{\sigma}_{A^{\prime}} \hat{\varrho} \hat{\sigma}_{A}^{\dagger}\right)\right.} \\
& +\delta_{A A^{\prime *}}^{+}\left(\hat{\sigma}_{A} \hat{\sigma}_{A^{\prime}}^{\dagger} \hat{\varrho}-\hat{\sigma}_{A^{\prime}}^{\dagger} \hat{\varrho} \hat{\sigma}_{A}\right) \\
& +\delta_{A A^{\prime *}}^{-}\left(\hat{\sigma}_{A} \hat{\varrho} \hat{\sigma}_{A^{\prime}}^{\dagger}-\hat{\varrho} \hat{\sigma}_{A^{\prime}}^{\dagger} \hat{\sigma}_{A}\right) \\
& \left.\left.\left.+\delta_{A^{*} A^{\prime}}^{+}\left(\hat{\sigma}_{A}^{\dagger} \hat{\varrho} \hat{\sigma}_{A^{\prime}}-\hat{\varrho} \hat{\sigma}_{A^{\prime}} \hat{\sigma}_{A}^{\dagger}\right)\right]\right\} \hat{O}\right) .
\end{aligned}
$$

The relationship (18) obviously implies the relationship

$$
\operatorname{Tr}\left[\frac{\mathrm{d} \varrho(t)}{\mathrm{d} t} \hat{O}(0)\right]=\operatorname{Tr}\left[\hat{\varrho}(0) \frac{\mathrm{d} \hat{O}(t)}{\mathrm{d} t}\right] .
$$

Since $\hat{O}$ is an arbitrary system operator, from Eqs. (A19) and (A20) it then follows that Eq. (19) must be valid.

\section{APPENDIX B: DERIVATION OF Eq. (20)}

For weak atom-field coupling, the on-resonant term

$$
\begin{gathered}
\hat{F}^{\prime}(t)=\frac{i}{\hbar} \sum_{A} \int_{0}^{\prime \infty} \mathrm{d} \omega\left\{\left[\hat{O}(t), \hat{\mathbf{d}}_{A}(t)\right] \underline{\hat{\mathbf{E}}}\left(\mathbf{r}_{A}, \omega, t\right)\right. \\
\left.+\underline{\hat{\mathbf{E}}}^{\dagger}\left(\mathbf{r}_{A}, \omega, t\right)\left[\hat{O}(t), \hat{\mathbf{d}}_{A}(t)\right]\right\},
\end{gathered}
$$

in the Hamiltonian $\hat{\tilde{H}}_{S}$ in Eq. (A16) can also be treated within the approximation scheme outlined in Appendix A for the off-resonant term $\hat{F}^{\prime \prime}(t)$ [Eq. (A1)]. Writing $\hat{F}^{\prime}(t)$ as

$$
\hat{F}^{\prime}(t)=\hat{F}_{\text {free }}^{\prime}+\sum_{A, A^{\prime}} F_{A A^{\prime}}^{\prime}(t)
$$

where $\hat{F}_{\text {free }}^{\prime}$ is defined according to Eqs. (A7) and (A17), but with $\int_{0}^{\prime \infty} \mathrm{d} \omega \ldots$ in place of $\int_{0}^{\prime \prime \infty} \mathrm{d} \omega \ldots$, and repeating the steps leading to Eq. (A14), we obviously arrive at an equation for $\hat{F}_{A A^{\prime}}^{\prime}(t)$ which again looks like Eq. (A14), but again with $\int_{0}^{\prime \infty} \mathrm{d} \omega \ldots$ in place of $\int_{0}^{\prime \prime \infty} \mathrm{d} \omega \ldots$.. Note that the equation for $\hat{F}_{A A^{\prime}}^{\prime}(t)$ also applies to the case $A=A^{\prime}$. Since the frequency integrals now run over the resonance region, only the $\delta$-function parts of the $\zeta$ functions do effectively contribute to the frequency integrals, i.e., we may approximate the $\zeta$-functions by $\delta$ functions,

$$
\zeta\left(\omega \pm \tilde{\omega}_{A^{\prime}}\right) \rightarrow \pi \delta\left(\omega \pm \tilde{\omega}_{A^{\prime}}\right) .
$$

Inserting Eq. (B2) [together with the equation for $\hat{F}_{A A^{\prime}}^{\prime}(t)$ and Eq. (B3)] into the expression for $\hat{\tilde{H}}_{\mathrm{S}}$ in the first term on the right-hand side of Eq. (A16), we derive

$$
\begin{aligned}
\dot{\hat{O}}= & -\frac{1}{2} i \sum_{A} \tilde{\omega}_{A}\left[\hat{O}, \hat{\sigma}_{A z}\right]+\hat{F}_{\text {free }} \\
& -\frac{1}{2} \sum_{A, A^{\prime}}\left\{\Gamma_{A^{*} A^{\prime}}\left[\hat{O}, \hat{\sigma}_{A}^{\dagger}\right] \hat{\sigma}_{A^{\prime}}+\Gamma_{A A^{\prime *} \hat{\sigma}_{A^{\prime}}^{\dagger}}\left[\hat{O}, \hat{\sigma}_{A}\right]\right\} \\
& +i \sum_{A, A^{\prime}}^{\prime}\left\{\delta_{A^{*} A^{\prime}}^{-}\left[\hat{O}, \hat{\sigma}_{A}^{\dagger}\right] \hat{\sigma}_{A^{\prime}}+\delta_{A A^{\prime *}}^{+}\left[\hat{O}, \hat{\sigma}_{A}\right] \hat{\sigma}_{A^{\prime}}^{\dagger}\right. \\
& \left.+\delta_{A A^{\prime *}}^{-} \hat{\sigma}_{A^{\prime}}^{\dagger}\left[\hat{O}, \hat{\sigma}_{A}\right]+\delta_{A^{*} A^{\prime}}^{+} \hat{\sigma}_{A^{\prime}}\left[\hat{O}, \hat{\sigma}_{A}^{\dagger}\right]\right\},
\end{aligned}
$$

where $\Gamma_{A A^{\prime}}$ is given by Eq. (21), and

$$
\hat{F}_{\text {free }}=\hat{F}_{\text {free }}^{\prime}+\hat{F}_{\text {free }}^{\prime \prime} \text {. }
$$

Assuming that the medium-assisted electromagnetic free field is in the vacuum state, thus

$$
\left\langle\hat{F}_{\text {free }}\right\rangle=0,
$$

and recalling the procedure that has led from Eq. (A16) via (A19) to Eq. (19), we see that Eq. (B4) just leads to Eq. (20). 


\section{APPENDIX C: DERIVATION OF Eq. (77)}

To calculate $P_{B}(t)=\left|C_{B}(t)\right|^{2}$ in lowest-order of perturbation theory with regard to Eq. (33), we replace $C_{A}$ and $C_{B}$ on the right-hand side of the equation for $\dot{C}_{B}$ by their respective initial values 1 and 0 , and integrate both sides of the equation with respect to time to obtain

$$
\begin{aligned}
& C_{B}(t)=i \delta_{B^{*} A} \int_{0}^{t} \mathrm{~d} t^{\prime} e^{i\left(\tilde{\omega}_{B}-\tilde{\omega}_{A}\right) t^{\prime}} \\
&+\int_{0}^{t} \mathrm{~d} t^{\prime} \int_{0}^{t^{\prime}} \mathrm{d} t^{\prime \prime} \int_{0}^{\prime \infty} \mathrm{d} \omega K_{B^{*} A}\left(t^{\prime}, t^{\prime \prime} ; \omega\right) .
\end{aligned}
$$

Using Eq. (34) together with the relationship

$$
\begin{aligned}
& e^{-i \omega\left(t^{\prime}-t^{\prime \prime}\right)} \theta\left(t^{\prime}-t^{\prime \prime}\right) \\
& =-\frac{1}{2 \pi i} \int_{-\infty}^{\infty} \mathrm{d} \omega^{\prime} \frac{e^{-i \omega^{\prime}\left(t^{\prime}-t^{\prime \prime}\right)}}{\omega^{\prime}-\omega+i 0_{+}},
\end{aligned}
$$

we may rewrite Eq. (C1) as

$$
\begin{aligned}
& C_{B}(t)=i \delta_{B^{*} A} 2 \pi \delta^{(t)}\left(\tilde{\omega}_{A}-\tilde{\omega}_{B}\right) \\
& +\frac{2}{i \hbar \varepsilon_{0}} \int_{-\infty}^{\infty} \mathrm{d} \omega^{\prime} \int_{0}^{\prime \infty} \mathrm{d} \omega \frac{\omega^{2}}{c^{2}} \frac{\mathbf{d}_{B}^{*} \operatorname{Im} \boldsymbol{G}\left(\mathbf{r}_{B}, \mathbf{r}_{A}, \omega\right) \mathbf{d}_{A}}{\omega^{\prime}-\omega+i 0_{+}} \\
& \quad \times \delta^{(t)}\left(\omega^{\prime}-\tilde{\omega}_{B}\right) \delta^{(t)}\left(\tilde{\omega}_{A}-\omega^{\prime}\right),
\end{aligned}
$$

where

$$
\delta^{(t)}(\omega)=\frac{1}{2 \pi} \int_{0}^{t} \mathrm{~d} t^{\prime} e^{-i \omega t^{\prime}}
$$

The function $\Delta\left(\omega^{\prime}\right)=\delta^{(t)}\left(\omega^{\prime}-\tilde{\omega}_{B}\right) \delta^{(t)}\left(\tilde{\omega}_{A}-\omega^{\prime}\right)$ in Eq. (C3) is essentially different from zero in an interval around $\tilde{\omega}_{A} \simeq \tilde{\omega}_{B}$, the extension of which is of the order of magnitude of $1 / t$. For sufficiently long times $t$, it is reasonable to assume that the function

$$
R\left(\omega^{\prime}\right)=\int_{0}^{\prime \infty} \mathrm{d} \omega \frac{\omega^{2}}{c^{2}} \frac{\mathbf{d}_{B}^{*} \operatorname{Im} \boldsymbol{G}\left(\mathbf{r}_{B}, \mathbf{r}_{A}, \omega\right) \mathbf{d}_{A}}{\omega^{\prime}-\omega+i 0_{+}}
$$

is slowly varying on the frequency scale of variation of $\Delta\left(\omega^{\prime}\right)$. The integral over $\omega^{\prime}$ can then be performed separately to yield

$$
\int_{-\infty}^{\infty} \mathrm{d} \omega^{\prime} \delta^{(t)}\left(\omega^{\prime}-\tilde{\omega}_{B}\right) \delta^{(t)}\left(\tilde{\omega}_{A}-\omega^{\prime}\right)=\delta^{(t)}\left(\tilde{\omega}_{A}-\tilde{\omega}_{B}\right)
$$

and Eq. C3 takes the form of $(t \rightarrow \infty)$

$$
\begin{aligned}
& C_{B}(t)=\left[2 \pi i \delta_{B^{*} A}+\frac{2}{i \hbar \varepsilon_{0}}\right. \\
& \left.\times \int_{0}^{\prime \infty} \mathrm{d} \omega \frac{\omega^{2}}{c^{2}} \frac{\mathbf{d}_{B}^{*} \operatorname{Im} \boldsymbol{G}\left(\mathbf{r}_{B}, \mathbf{r}_{A}, \omega\right) \mathbf{d}_{A}}{\tilde{\omega}_{A}-\omega+i 0_{+}}\right] \delta^{(t)}\left(\tilde{\omega}_{A}-\tilde{\omega}_{B}\right) .
\end{aligned}
$$

To evaluate the frequency integral in Eq. (C7), we complete it by adding the corresponding off-resonant part and subsequently subtract it. The integral $\int_{0}^{\infty} \mathrm{d} \omega \ldots$ can then be approximately evaluated by extending the lower limit to $-\infty$ and using contour integral technique in a similar way as in Ref. [14]. The remaining integral $\int_{0}^{\prime \prime} \infty \mathrm{d} \omega \ldots$ can again be treated as a principal-value integral to give $-2 \pi i \delta_{B^{*} A}^{-}$[cf. Eq. (14)], which (apart from the quantum correction) cancels out the first term in Eq. (C7). Taking into account that for $t \rightarrow \infty,\left|\delta^{(t)}(\omega)\right|^{2}$ behaves like

$$
\left|\delta^{(t)}(\omega)\right|^{2}=\frac{t}{2 \pi} \delta(\omega)
$$

we eventually arrive at

$$
\begin{aligned}
P_{B}(t) & =\left|C_{B}(t)\right|^{2}=\frac{2 \pi}{\hbar^{2}}\left(\frac{\tilde{\omega}_{A}^{2}}{\varepsilon_{0} c^{2}}\right)^{2} \\
\times & \left|\mathbf{d}_{B}^{*} \boldsymbol{G}\left(\mathbf{r}_{B}, \mathbf{r}_{A}, \tilde{\omega}_{A}\right) \mathbf{d}_{A}\right|^{2} \delta\left(\tilde{\omega}_{A}-\tilde{\omega}_{B}\right) t,
\end{aligned}
$$

which together with Eq. (37) leads to Eq. (77).

\section{APPENDIX D: DERIVATION OF Eqs. (88) and (91)}

Let us briefly outline the derivation of Eqs. (88) and (91) (for more details, see [15]). In the Schrödinger picture, the two-time correlation function in Eq. (85) can be given by

$$
\begin{aligned}
& \left\langle\hat{\mathbf{E}}^{(-)}\left(\mathbf{r}, t_{2}\right) \hat{\mathbf{E}}^{(+)}\left(\mathbf{r}, t_{1}\right)\right\rangle \\
& =\left\langle\psi\left(t_{2}\right)\left|\hat{\mathbf{E}}^{(-)}(\mathbf{r}) e^{-i \hat{H}_{\text {eff }}\left(t_{2}-t_{1}\right) / \hbar} \hat{\mathbf{E}}^{(+)}(\mathbf{r})\right| \psi\left(t_{1}\right)\right\rangle .
\end{aligned}
$$

Calculating it by using Eqs. (30), (32), (86), (87), and (4), and inserting the resulting expression into Eq. (85), we derive

$$
\begin{aligned}
& S\left(\mathbf{r}, \omega_{\mathrm{S}}, T\right)=\mid \sum_{A} \int_{0}^{T} \mathrm{~d} t_{1}\left[e^{i\left(\omega_{\mathrm{S}}-\tilde{\omega}_{A}\right) t_{1}} \int_{0}^{t_{1}} \mathrm{~d} t^{\prime} C_{A}\left(t^{\prime}\right)\right. \\
& \left.\times \int_{0}^{\prime \infty} \mathrm{d} \omega \frac{\omega^{2}}{\pi \epsilon_{0} c^{2}} \operatorname{Im} \boldsymbol{G}\left(\mathbf{r}, \mathbf{r}_{A}, \omega\right) \mathbf{d}_{A} e^{-i\left(\omega-\tilde{\omega}_{A}\right)\left(t_{1}-t^{\prime}\right)}\right]\left.\right|^{2} .
\end{aligned}
$$

For the two-atom system under consideration, in the weak-coupling regime, $C_{A(B)}\left(t^{\prime}\right)$ may be replaced with $C_{A(B)}\left(t_{1}\right)$, where $C_{A(B)}\left(t_{1}\right)$ is given according to Eqs. (38) and (39),

$$
\begin{aligned}
& C_{A(B)}(t)=\frac{1}{2} \\
& \quad \times\left[e^{\left(-\Gamma_{+} / 2+i \delta_{A^{*} B}\right) t}+(-) e^{\left(-\Gamma_{-} / 2-i \delta_{A^{*} B}\right) t}\right] .
\end{aligned}
$$


$\left(\Gamma_{A^{*} A}=\Gamma_{B^{*} B}\right.$ and $\left.\mathcal{K}_{A^{*} B}=\mathcal{K}_{B^{*} A}\right)$. The $t^{\prime}$-integral may then be regarded, in the long-time limit, as being $\zeta\left(\tilde{\omega}_{A^{\prime}}-\omega\right)$, so that the factor $\mathbf{F}_{A^{\prime}}$ [Eq. (90)] can be put in front of the $t_{1}$-integral. Now the $t_{1}$-integral can be performed to obtain Eq. (88) $(T \rightarrow \infty)$.

For strong atom-field coupling, we use Eq. (45) and express in Eq. (D2) $C_{A(B)}(t)$ in terms of $C_{ \pm}(t)$,

$$
\begin{gathered}
S\left(\mathbf{r}, \omega_{\mathrm{S}}, T\right)=\frac{1}{2} \mid \int_{0}^{T} \mathrm{~d} t_{1} e^{i\left(\omega_{\mathrm{S}}-\tilde{\omega}_{A}\right) t_{1}} \\
\times\left\{\int_{0}^{t_{1}} \mathrm{~d} t^{\prime} C_{+}\left(t^{\prime}\right) e^{i \delta_{A^{*} B} t^{\prime}} \int_{0}^{\prime \infty} \mathrm{d} \omega \frac{\omega^{2}}{\pi \epsilon_{0} c^{2}}\right. \\
\times\left[\operatorname{Im} \boldsymbol{G}\left(\mathbf{r}, \mathbf{r}_{A}, \omega\right) \mathbf{d}_{A}+\operatorname{Im} \boldsymbol{G}\left(\mathbf{r}, \mathbf{r}_{B}, \omega\right) \mathbf{d}_{B}\right] \\
\times e^{-i\left(\omega-\tilde{\omega}_{A}\right)\left(t_{1}-t^{\prime}\right)} \\
+\int_{0}^{t_{1}} \mathrm{~d} t^{\prime} C_{-}\left(t^{\prime}\right) e^{-i \delta_{A^{*} B^{\prime}} t^{\prime}} \int_{0}^{\infty} \mathrm{d} \omega \frac{\omega^{2}}{\pi \epsilon_{0} c^{2}} \\
\times\left[\operatorname{Im} \boldsymbol{G}\left(\mathbf{r}, \mathbf{r}_{A}, \omega\right) \mathbf{d}_{A}-\operatorname{Im} \boldsymbol{G}\left(\mathbf{r}, \mathbf{r}_{B}, \omega\right) \mathbf{d}_{B}\right] \\
\left.\times e^{-i\left(\omega-\tilde{\omega}_{A}\right)\left(t_{1}-t^{\prime}\right)}\right\}\left.\right|^{2} .
\end{gathered}
$$

Recall that either the state $|+\rangle$ or the state $|-\rangle$ is strongly coupled to the medium-assisted electromagnetic field, but not both at the same time. The state which is weakly coupled to the field can be treated in the same way as above. For the strongly coupled state, we again assume a Lorentzian shape for the field resonance $\left(\omega_{m}\right.$, central frequency; $\Delta \omega_{m}$, width; cf. Section IVB and evaluate the $\omega$-integral. Taking $C_{ \pm}\left(t^{\prime}\right)$ from Eq. (53), we then can evaluate the remaining time integrals to obtain Eq. (91) together with Eq. (92) $(T \rightarrow \infty)$.

* On leave from the Institute of Physics, National Center for Natural Sciences and Technology, 1 Mac Dinh Chi Street, District 1, Ho Chi Minh city, Vietnam.

[1] M. D. Lukin and P. R. Hemmer, Phys. Rev. Lett. 84, 2818 (2000).

[2] A. Barenco, D. Deutsch, A. Ekert, and R. Jozsa, Phys. Rev. Lett. 74, 4083 (1995).

[3] S. John and J. Wang, Phys. Rev. B 43, 12772 (1991); G. Kweon and N. M. Lawandy, J. Mod. Opt. 41, 311 (1994); S. John and T. Quang, Phys. Rev. A 52, 4083 (1995); S. Bay, P. Lambropoulos, and K. Mølmer, ibid. 55, 1485 (1997).

[4] T. Kobayashi, Q. Zheng, and T. Sekiguchi, Phys. Rev. A 52, 2835 (1995).

[5] A. Takada and K. Ujihara, Opt. Commun. 160, 146 (1999).

[6] G. Juzeliūnas and D. L. Andrews, Phys. Rev. B 50, 13371 (1994).
[7] G. S. Agarwal and S. D. Gupta, Phys. Rev. A 57, 667 (1998).

[8] Ho Trung Dung, S. Scheel, L. Knöll, and D.-G. Welsch, J. Opt. B: Quant. Semiclass. Opt. 4, S169 (2002).

[9] E. V. Goldstein and P. Meystre, Phys. Rev. A 56, 5135 (1997).

[10] G. Kurizki and A. Z. Genack, Phys. Rev. Lett. 61, 2269 (1988).

[11] Q. Zheng, T. Kobayashi, and T. Sekiguchi, Phys. Rev. Lett. 77, 406 (1996); G. Kurizki, A. G. Kofman, and A. Z. Genack, ibid. 77, 407 (1996).

[12] G. Kurizki, A. G. Kofman, and V. Yudson, Phys. Rev. A 53, R35 (1996).

[13] L. Knöll, S. Scheel, and D.-G. Welsch, in Coherence and Statistics of Photons and Atoms, edited by J. Peřina (John Wiley \& Son, New York, 2001), p. 1.

[14] Ho Trung Dung, L. Knöll, and D.-G. Welsch, Phys. Rev. A 65, 043813 (2002).

[15] S. Scheel, L. Knöll, and D.-G. Welsch, Phys. Rev. A 60, 4094 (1999); Ho Trung Dung, L. Knöll, and D.-G. Welsch, ibid. 62, 053804 (2000).

[16] R. H. Lehmberg, Phys. Rev. A 2, 889 (1970).

[17] J. R. Ackerhalt, P. L. Knight, and J. H. Eberly, Phys. Rev. Lett. 30, 456 (1973); J. R. Ackerhalt and J. H. Eberly, Phys. Rev. D 10, 3350 (1974).

[18] G. S. Agarwal, Quantum Optics, Vol. 70 of Springer Tracts in Modern Physics (Springer, Berlin, 1974).

[19] A. Beige and G. C. Hegerfeldt, Phys. Rev. A 59, 2385 (1999).

[20] C. Skornia, J. von Zanthier, G. S. Agarwal, E. Werner, and H. Walther, Phys. Rev. A 64, 053803 (2001).

[21] J. M. Wylie and J. E. Sipe, Phys. Rev. A 32, 2030 (1985); J. P. Dowling, Found. Phys. 28, 855 (1998).

[22] M. Tavis and F. W. Cummings, Phys. Rev. 170, 379 (1968).

[23] T. Förster, in Modern Quantum Chemistry, edited by O. Sinagoglu (Academic, New York, 1965).

[24] T. Förster, Ann. Phys. (Leipzig) 1, 55 (1948); D. L. Dexter, J. Chem. Phys. 21, 836 (1953).

[25] W. Vogel, D.-G. Welsch, and S. Wallentowitz, Quantum Optics, An Introduction (Wiley-VCH, Berlin, 2001).

[26] T. G. Rudolph, Z. Ficek, and B. J. Dalton, Phys. Rev. A 52, 636 (1995). 